\title{
ON THREE-DIMENSIONAL CAUCHY-RIEMANN MANIFOLDS
}

\author{
LÁSZLÓ LEMPERT
}

1. Introduction. In this paper we shall be concerned with three-dimensional strictly pseudoconvex Cauchy-Riemann (CR) manifolds. The prime examples of such manifolds arise as smooth boundaries $M$ of bounded strictly pseudoconvex domains $D \subset \mathbb{C}^{2}$. For any point $p \in M$ the tangent space $T_{p} M$ contains a codimension one subspace $H_{p} M$ which is invariant under the action of the almost complex tensor of $\mathbb{C}^{2}$ ("multiplication by $i$ "). The collection of these subspaces $H_{p} M$ forms a bundle $H M \subset T M$ called the horizontal or contact bundle. (Indeed, strict pseudoconvexity of $M$ implies that the plane field $\left\{H_{p} M\right\}$ is nondegenerate, i.e. defines a contact structure.) The almost complex tensor of $\mathbb{C}^{2}$ then restricts to a bundle endomorphism $J: H M \rightarrow H M$ such that $J^{2}=-\mathrm{id}$. The bundle $H M \subset T M$ together with this endomorphism $J$ defines the CR structure of $M$.

Slightly more generally, let $\bar{D}=D \cup M$ be a four dimensional compact manifold with boundary $M$, interior $D$, endowed with a smooth almost complex tensor which is integrable on $D$. If $\bar{D}$ is strictly pseudoconvex-i.e., in a neighborhood of any $p \in M$ there is a smooth strictly plurisubharmonic function $u$, negative on $D, 0$ on $M$, but $d u \neq 0$ on $M$-then $M$ inherits a strictly pseudoconvex CR structure from $\bar{D}$. In this case we shall say that $M$ bounds a strictly pseudoconvex surface.

This leads us to the abstract definition. A strictly pseudoconvex (threedimensional) CR manifold is a compact manifold $M$ (without boundary), $\operatorname{dim} M=3$, endowed with a contact structure $H M=\left\{H_{p} M: p \in M\right\} \subset T M$ and an endomorphism $J$ of $H M$ such that $J^{2}=-\mathrm{id}$. (Throughout this paper unless otherwise stated we shall be working with infinitely differentiable objects. Thus $M, H M, J$ are assumed to be smooth in this sense.) Furthermore, a differentiable function $f: M \rightarrow \mathbb{C}$ is a $\mathrm{CR}$ function if for any $p \in M$ the restriction

$$
\left.d f\right|_{H_{p} M}: H_{p} M \rightarrow T_{f(p)} \mathbb{C}
$$

intertwines the action of $J$ on $H_{p} M$ on the one hand and the action of the almost complex tensor of $\mathbb{C}$ on $T_{f(p)} \mathbb{C}$ on the other.

A basic problem of the theory of CR manifolds is to decide when an abstract strictly pseudoconvex CR manifold $M$ is the boundary of some strictly

Received by the editors September 20, 1991.

1991 Mathematics Subject Classification. Primary 32F40, 32 G07.

Research supported by an NSF grant. 
pseudoconvex manifold. By theorems of Harvey-Lawson and Kohn, and also resolution of singularities, this happens if and only if there is an embedding of $M$ into some Euclidean space $\mathbb{C}^{k}$ whose components are CR functions. Also, by theorems of Boutet de Monvel, Burns, and Kohn this happens if and only if the range of a certain differential operator associated with $M, \square_{b}$, has closed range; see $[5,6,12,17,18]$.

The corresponding questions can be asked for strictly pseudoconvex CR manifolds of any (odd) dimension, and for dimension at least 5 the answer, namely "always", has been given by Boutet de Monvel (see [5]). In contrast, in the three-dimensional case examples by Rossi and Nirenberg and finally a theorem of Jacobowitz-Treves show that the answer is "almost never"; see [13, 29,32].

Unfortunately, the above sufficient and necessary conditions for $M$ to bound a strictly pseudoconvex surface $\bar{D}$ are not completely satisfactory in that given an abstract CR manifold these conditions are rarely verifiable. This may not be our fault; perhaps it is a manifestation of how deep a property it is for an $M$ to bound a surface $\bar{D}$. The inherent difficulties in distinguishing between the bounding and nonbounding cases are demonstrated by Rossi's example, which does not bound, but admits a quotient by a $\mathbb{Z} / 2$ action which does bound.

These difficulties notwithstanding, one would like to be able to answer simple questions about the bounding property (or equivalently: embeddability) like the following two:

1. Suppose $M_{k}$ is a sequence of CR structures on a fixed smooth manifold converging to a CR structure $M$. If each $M_{k}$ is embeddable, does it follow that so is $M$ ?

2. Suppose $M_{0} \subset \mathbb{C}^{n}$ is an embedded $\mathrm{CR}$ manifold and $M$ is a small perturbation of the CR structure of $M_{0}$, which is also embeddable. Does it follow that $M$ is embeddable as a submanifold of $\mathbb{C}^{n}$, close to $M_{0}$ ? ing.

Of course, "convergence" and "perturbation" have to be given a precise mean-

The first question was first asked by Burns in [6]. The second was asked by Burns and Epstein in [7], where they solved a problem weaker than the one raised in 1. Namely, they proved that in the space of all CR structures on a given smooth manifold the embeddable ones form an $F_{\sigma}$ set. (Question 1 asks if this set is closed.) The results of [7] also show that questions 1 and 2 are related. Question 2 was asked by Cheng and Lee as well, cf. [9], who explained the importance of this question for constructing moduli spaces of embeddable CR structures.

The aim of this work is to approach the problems of embeddability through one dimensional group actions. We shall see that in certain situations $M$ is embeddable if and only if it admits a certain type of circle action. While this is not a completely sufficient and necessary condition, and moreover the existence of such a circle action may not be easily verifiable, we can use this approach to give an affirmative answer to question 2 above, at least in the case when $M_{0}$ is a strictly convex hypersurface in $\mathbb{C}^{2}$. By the results of [7] this then also implies that the answer to question 1 is positive, provided $M_{k}$ and $M$ are small perturbations of an $M_{0}$ that can be embedded as a strictly convex hypersurface in $\mathbb{C}^{2}$. 
The main results are stated in $\S 4$. They are formulated in terms of notions defined in $\S 3$.

After this manuscript had been completed, we received a preprint from C. Epstein, in which-among other things-he proves embeddability of certain three-dimensional CR manifolds, extending some results from [7]. This result can be shown to be equivalent to our Theorem 4.1 below (in the case when the $S^{1}$ action is free, and the "Beltrami coefficients" of the action are close to constant.)

2. CR actions. In this paper $M$ will always denote a connected smooth (meaning infinitely differentiable), compact strictly pseudoconvex $\mathrm{CR}$ manifold of dimension 3. The CR structure is given by the contact bundle $H M \subset T M$ and the endomorphism $J: H M \rightarrow H M, J^{2}=-\mathrm{id}$. Such a manifold can always be given a canonical orientation as follows. Let $X$ be a nonvanishing local section of $H M$. By declaring the frame $X, J X,[J X, X]$ positive, we obtain a consistent orientation of $M$.

Now let $\left\{g_{t}: t \in \mathbb{R}\right\}$ be a smooth $\mathbb{R}$-action on $M$. It is called transverse if the infinitesimal generator of this action, $\left(d g_{t} / d t\right)_{t=0}$, which is a smooth vector field, is everywhere transverse to the contact plane field $\left\{H_{p} M\right\}$. Transverse $\mathbb{R}$-actions fall into two classes according to whether for a (or any) nonvanishing local section $X$ of $H M$ the frame $X, J X$ and the infinitesimal generator $\left(d g_{t} / d t\right)_{t=0}$ is positively oriented or not. In the former case the action will be called positive.

In this section we shall consider CR actions $\left\{g_{t}\right\}$, i.e., such that for any $t$ the differential $g_{t *}$ preserves the contact plane field $\left\{H_{p} M\right\}$ and also the action of $J$ on $H M$. Baouendi-Rothschild-Treves (see [1]) proved that the existence of a local transverse CR action implies local embeddability. The following is a global version of their result.

Theorem 2.1. Suppose that $M$ admits a smooth $C R$ action of $\mathbb{R}$, which is transverse. Then $M$ is the boundary of a strictly pseudoconvex complex surface (i.e., it is embeddable).

Let us point out that transversality here cannot be dispensed with: an example of Barrett (see [2]) shows this. Rossi's example also admits a nontransversal circle action. In fact, in Barrett's nonembeddable example the CR manifold admits a CR torus action, which is transverse in an obvious sense. However, no one-dimensional sub-action exists which itself would be transverse.

We shall not prove this theorem here. For the moment we content ourselves with the following simple lemma which relates $\mathbb{R}$-actions to compact group actions.

Lemma 2.2. Suppose that $M$ admits a smooth $C R$ action $\left\{g_{t}: t \in \mathbb{R}\right\}$ of $\mathbb{R}$, which is transverse. Then this action is quasiperiodic; i.e., there exist a torus $T^{k}$, a smooth $C R$ action $\left\{h_{u}: u \in T^{k}\right\}$ of it on $M$, and a homomorphism $\varphi: \mathbb{R} \rightarrow T^{k}$ with dense image such that $g_{t}=h_{\varphi(t)}$. 
Proof. Let $V$ be the infinitesimal generator of $\left\{g_{t}\right\}$, and let $\alpha$ be the (contact) 1 -form defined by

$$
\left.\alpha\right|_{H_{p} M}=0, \quad\langle\alpha, V\rangle=1
$$

Then the Lie derivative of $\alpha$ with respect to the field $V$ is

$$
\left.\left.L_{V} \alpha=d\langle\alpha, V\rangle+V\right\lrcorner d \alpha=V\right\lrcorner d \alpha .
$$

On the other hand, the flow of $V$ preserves the plane field $\left\{H_{p} M\right\}$, so that

$$
\operatorname{ker} L_{V} \alpha \supset \operatorname{ker} \alpha=\left\{H_{p} M\right\} .
$$

Since (2.1) implies that also $V \in \operatorname{ker} L_{V} \alpha$, we infer that $L_{V} \alpha \equiv 0$ and hence $L_{V} d \alpha \equiv 0$.

Now we can define a metric on $M$ which is invariant under the action. Choose a vector $X \in H_{p} M$ so that

$$
\langle d \alpha, X \wedge J X\rangle=1 \text {. }
$$

By declaring $X, J X, V_{p}$ an orthonormal frame, we obtain a Riemann metric on $M$, which, in view of the $\left\{g_{t}\right\}$ invariance of $d \alpha$ and $J$, itself is $\left\{g_{t}\right\}$ invariant. (The construction of this invariant metric is due to Tanaka [35].)

Thus the one-parameter group $\left\{g_{t}\right\}$ appears as a subgroup of isometries of a compact Riemannian manifold. Such isometry groups are compact. The closure of $\left\{g_{t}\right\}$ in the full isometry group is a compact connected Abelian group, hence isomorphic to a torus $T^{k}$. Since any isometry $h_{u}$ corresponding to an element $u \in T^{k}$ is the limit (in the $C^{\infty}$ topology) of a sequence of type $g_{t_{\nu}}$, we obtain that $T^{k}$ acts by $\mathrm{CR}$ automorphisms.

Notice that arbitrarily near to $V$ there are vector fields that generate a periodic sub-action of the $T^{k}$-action. Hence it follows that for $M$ as in Lemma 2.2 there even exists a transverse CR $S^{1}$-action. Thus Theorem 2.1 will be a consequence of Theorem 4.1 below.

3. Inner and outer actions. Obviously it is very exceptional for a CR manifold (embeddable or not) to admit CR automorphisms, let alone nontrivial CR actions of $\mathbb{R}$. In this section we shall introduce a type of action, more general than CR actions, the existence of which in a sense characterizes CR manifolds that are boundaries.

Let us start with a contact action of the circle $S^{1}$ which is positive in the sense explained in $\S 2$. We shall think of $S^{1}$ as the unit circle in the complex plane $\mathbb{C}$. Denote this action $\left\{g_{\zeta}: \zeta \in S^{1}\right\}$. Along orbits of the action we shall measure how far the action is from being $\mathrm{CR}$ as follows. Let $p \in M$ and $X \in \mathbb{C} \otimes H_{p} M$ such that $X$ and $\bar{X}$ are linearly independent. Since $\operatorname{dim}_{\mathbb{C}} \mathbb{C} \otimes H_{p} M=2$, this means that $X$ and $\bar{X}$ span $\mathbb{C} \otimes H_{p} M$ so that there are numbers $\alpha, \beta \in \mathbb{C}$, not both zero, such that

$$
\alpha \bar{X}+\beta X \in H_{p}^{0,1} M
$$

$H_{p}^{0,1} M$ meaning the space of $(0,1)$ vectors, i.e., of the form $i Y-J Y, Y \in$ $H_{p} M .|\alpha| \neq|\beta|$, for otherwise the $(0,1)$ vector in $(3.1)$ and its conjugate 
would be dependent. Hence either $|\alpha|<|\beta|$ or $|\alpha|>|\beta|$. We shall assume that $X$ is chosen so the $|\alpha|>|\beta|$ holds (as, e.g., when $\bar{X}$ itself is a $(0,1)$ vector). In this case we shall say that $X$ is a $(1,0)$-like vector, while $\bar{X}$ is a $(0,1)$-like vector.

Now fix $p$ and $X$ and push forward $X$ by a diffeomorphism $g_{\zeta}$ for some $\zeta \in S^{1}$. Since $g_{\zeta}$ preserves the contact bundle $H M, g_{\zeta *} X, g_{\zeta *} \bar{X} \in \mathbb{C} \otimes$ $H_{g_{\zeta}(p)} M$, and these two vectors are again independent. Therefore there are complex numbers $a, b$, not both of them 0 , such that

$$
a g_{\zeta *} \bar{X}+b g_{\zeta *} X \in H_{g_{\zeta}(p)}^{0,1} M .
$$

Although $a, b$ are not unique, $b / a=\mu(\zeta) \in \mathbb{C} \cup\{\infty\}$ is uniquely determined, and depends smoothly on $\zeta$. Since $|\mu(\zeta)| \neq 1$, as before, and $\mu(1)=\beta / \alpha$, it follows that

$$
|\mu(\zeta)|<1 \quad\left(\zeta \in S^{1}\right)
$$

$\mu$ will be called a Beltrami differential associated with the trajectory of $p$.

Strictly speaking, $\mu$ depends not only on the trajectory of $p$ but on $p$ itself and the vector $X$. However, this dependence is very simple:

Proposition 3.1. Let $p^{\prime}=g_{\omega}(p)$ be another point on the trajectory of $p\left(\omega \in S^{1}\right)$ and let $\mu^{\prime}(\zeta)$ denote the Beltrami differential determined by $p^{\prime}$ and a vector $X^{\prime} \in \mathbb{C} \otimes H_{p^{\prime}} M,\left|\mu^{\prime}(\zeta)\right|<1$. Then there exists a holomorphic automorphism $\varphi$ of the unit disc $\Delta \subset \mathbb{C}$ such that

$$
\mu^{\prime}(\zeta)=\varphi(\mu(\omega \zeta))
$$

Proof. Let us first observe that if $a$ and $b$ are complex numbers, then the fractional linear transformation of $\mathbb{C}$ given by

$$
s \mapsto \frac{\bar{a} s+b}{a+\bar{b} s}
$$

maps the unit disc into itself if $|a|>|b|$; into its complement if $|a|<|b|$; and into the unit circle if $|a|=|b|$. Furthermore, in the first case the mapping is an automorphism of the disc.

Next, for the sake of brevity, introduce the notation

$$
X(\zeta)=g_{\zeta *} X, \quad X^{\prime}(\zeta)=g_{\zeta *} X^{\prime}
$$

Then there are complex numbers $a, b$ such that

$$
\bar{X}^{\prime}(1)=a \overline{X(\omega)}+b X(\omega)
$$

Since the fields $X(\zeta), X^{\prime}(\zeta)$ are invariant under the group action it follows that for any $\zeta \in S^{1}$

$$
\overline{X^{\prime}(\zeta)}=a \overline{X(\omega \zeta)}+b X(\omega \zeta)
$$

According to the definition of $\mu^{\prime}(\zeta)$ the following vector is of type $(0,1)$ :

$$
\overline{X^{\prime}(\zeta)}+\mu^{\prime}(\zeta) X^{\prime}(\zeta)=\left(a+\mu^{\prime}(\zeta) \bar{b}\right) \overline{X(\omega \zeta)}+\left(\mu^{\prime}(\zeta) \bar{a}+b\right) X(\omega \zeta)
$$

Hence

$$
\mu(\omega \zeta)=\left(\bar{a} \mu^{\prime}(\zeta)+b\right) /\left(a+\bar{b} \mu^{\prime}(\zeta)\right)
$$


Since $|\mu(\omega \zeta)|<1$ and $\left|\mu^{\prime}(\zeta)\right|<1$, the preliminary remark now implies the proposition.

It is easy to see that the action $\left\{g_{\zeta}\right\}$ is a CR action if and only if the Beltrami differentials associated with all trajectories are constant. We shall now introduce a weaker condition on the Beltrami differentials.

Definition 3.2. Suppose that a positive contact action $\left\{g_{\zeta}: \zeta \in S^{1}\right\}$ on $M$ has the property that all Beltrami differentials $\mu(\zeta)$ associated with its trajectories extend to a smooth function on the closure $\bar{\Delta}$ of the unit disc in such a way that this extension is holomorphic on $\Delta$. Then we shall call the action an inner action.

Analogously, if the Beltrami differentials extend to smooth functions on $\mathbb{P}_{1} \backslash \Delta$, holomorphic on $\mathbb{P}_{1} \backslash \bar{\Delta}$, we shall call the action outer. (Here $\mathbb{P}_{1}$ stands for the Riemann sphere.)

For example CR actions are both inner and outer, and the converse is also true.

Some remarks are in order here. First, although their definitions are very symmetric, inner and outer actions are essentially different. For example, inner actions rarely exist, and even if a given manifold $M$ admits an inner action, there can only be very few such actions (the parameter space is finite dimensional). On the other hand outer actions very often exist and on many manifolds $M$ their parameter space is a function space. For more precise statements see Theorem 6.4 and Remark 8.3.

The second remark we want to make here is that, in view of Proposition 3.1 and the maximum principle, to check that an action is inner (or outer) one needs to consider only one Beltrami differential for each orbit; if this has the required extendability property then so do all other Beltrami differentials associated with the given orbit.

4. On the existence of inner and outer $S^{1}$-actions. It turns out that the existence of inner $S^{1}$-actions on a given $M$ is related to the fact that $M$ is a boundary.

Theorem 4.1. If $M$ admits an inner $S^{1}$-action then $M$ is the boundary of a strictly pseudoconvex surface.

This theorem is related to Theorem 5.3 of [7]. Indeed, it is easy to see that with notation of that paper, $\phi \in E_{0}$ implies that the standard $S^{1}$ action on $S^{3} \subset \mathbb{C}^{2}$ is inner for the CR structure $W^{\phi}$ (and conversely). Hence, from our theorem above it follows that this $\mathrm{CR}$ structure embeds into some $\mathbb{C}^{n}$. Theorem 5.3 of [7] covers only the case when $W^{\phi}$ is a small deformation of the standard structure of $S^{3}$, but then it asserts more: namely that $W^{\phi}$ embeds into $\mathbb{C}^{2}$.

Theorem 4.1 has a partial converse. The converse involves the notion of strict linear convexity.

Definition 4.2. A smoothly bounded, bounded domain $D \subset \mathbb{C}^{2}$ is strictly linearly convex if for each boundary point $p \in \partial D$ there is a complex affine line $L$ through $p$ which does not intersect $\bar{D} \backslash\{p\}$ and has precisely firstorder contact with $\partial D$ at $p$, in the sense that with some $c>0 \operatorname{dist}(q, L) \geq$ 
$c \operatorname{dist}^{2}(q, p), q \in D$. In particular, strictly convex domains are strictly linearly convex, and any strictly linearly convex domain is strictly pseudoconvex.

Theorem 4.3. If $M$ is the boundary of a strictly linearly convex domain $D \subset \mathbb{C}^{2}$ then $M$ admits both inner $S^{1}$-action and outer $S^{1}$-actions.

These two theorems suggest that at least for an open set in the space of all CR manifolds, admitting an inner $S^{1}$-action and being the boundary of a strictly pseudoconvex complex surface are equivalent. To make such a statement precise, we shall discuss perturbations of a given (three-dimensional, compact) strictly pseudoconvex CR manifold $M_{0}$. We shall say that a strictly pseudoconvex CR manifold $M$ is a small perturbation of $M_{0}$ if $M$ and $M_{0}$ have the same underlying smooth manifold; also the contact bundles $H M$ and $H M_{0}$ are the same, and the almost complex tensors $J: H M \rightarrow H M$ and $J_{0}: H M_{0} \rightarrow H M_{0}$ are at distance less than $\varepsilon$, measured in the $C^{k}$-norm on tensors (relative to some Riemann metric on the underlying smooth manifold of $M$ and $M_{0}$ ). When a statement on $M$ is phrased as to hold for (sufficiently) small perturbations of $M_{0}$, by this we mean, as usual, that for some positive $\varepsilon$ and $k$, whenever the above-mentioned distance is less than $\varepsilon$, the statement on $M$ holds true.

A seemingly more general notion of perturbation is obtained if the contact structure is also allowed to change. However, small perturbations of a contact structure on a compact manifold are always equivalent to the initial structure so that, after all, our version of perturbation in essence is no more restrictive than the seemingly more general one.

Theorem 4.4. Let $M_{0} \subset \mathbb{C}^{2}$ be the boundary of a strictly linearly convex domain. If $M$ is a sufficiently small perturbation of $M_{0}$, the following two statements are equivalent:

(a) $M$ is the boundary of a strictly pseudoconvex complex surface.

(b) $M$ admits an inner action.

That (b) implies (a) is the content of Theorem 4.1. The converse follows from Theorem 4.3 and the following theorem, which shows that for embeddable CR manifolds the embeddings can be chosen in a stable way.

Theorem 4.5. Let $M_{0} \subset \mathbb{C}^{2}$ be the boundary of a strictly linearly convex domain and let $\delta, \ell$ be positive numbers. Suppose $M$ is a small perturbation of $M_{0}$ which is the boundary of a strictly pseudoconvex complex surface (i.e., it is embeddable into some $\mathbb{C}^{n}$ ). How small the perturbation should be depends on $\delta$ and $\ell$. Then $M$ admits a $C R$ embedding into $\mathbb{C}^{2}$ whose $C^{\ell}$ distance from the embedding $M_{0} \subset \mathbb{C}^{2}$ is less than $\delta$. In particular, if $\ell \geq 2$ and $\delta$ is sufficiently small, $M$ can be $C R$ embedded into $\mathbb{C}^{2}$ as the boundary of a strictly linearly convex domain.

Corresponding stability results for embeddings are known for strictly pseudoconvex CR manifolds of dimension at least 5; see Tanaka [35]. Tanaka's stability theorem holds for an initial CR structure $M_{0}$ with a certain cohomology group 0 , whereas we had to assume that $M_{0}$ admits a special embedding. 
Of course, the three-dimensional case is unique for-as said before-only in this dimension are there nonembeddable strictly pseudoconvex CR manifolds. Also in this dimension the relevant cohomology group is infinite dimensional.

The following theorem underscores the difference between inner and outer actions:

Theorem 4.6. Let $M_{0} \subset \mathbb{C}^{2}$ be the boundary of a strictly linearly convex domain, and $M$ a small perturbation of $M_{0}$. Then $M$ admits an outer action of $S^{1}$.

Let us remark here that "Beltrami coefficients" on CR manifolds have first been introduced by Korányi and Reimann. Among other things, they prove an embeddability result for certain types of Beltrami coefficients. Although our Beltrami differentials are in spirit related to theirs, there is no logical relationship, and they are different kinds of objects; see [19,20].

5. Proof of Theorem 4.1. Let $M$ admit an inner action $\left\{g_{\zeta}: \zeta \in S^{1}\right\}$. Then this action has no fixed points. Furthermore, by passing to a quotient by a finite subgroup we can and we shall assume that the action is effective; i.e., no $g_{\zeta}$ with $\zeta \neq 1$ is the identity $M \rightarrow M$.

Fixed point free and effective $S^{1}$-actions on three-dimensional compact manifolds are known to have a very simple structure. Their local model is the following. Consider an open disc $U \subset \mathbb{C}$ centered at the origin, a positive integer $k$ and a primitive $k$ th root of $1, \rho$. On $U \times S^{1}$ the cyclic group $\mathbb{Z} / k$ acts by

$$
(u, s) \mapsto\left(u \rho^{j}, s e^{2 \pi i j / k}\right), \quad j \in \mathbb{Z} / k .
$$

Since the action has no fixed points, the factor of $U \times S^{1}$ by this action is a smooth manifold $N$. The action of $S^{1}$ on $U \times S^{1}$

$$
(u, s) \mapsto(u, \zeta s) \quad\left(\zeta \in S^{1}\right)
$$

factors through the action (5.1) to define an $S^{1}$-action on $N$.

In fact, $N$ itself is diffeomorphic to $U \times S^{1}$, and the $S^{1}$ action on $N$ is equivalent to an $S^{1}$-action on $U \times S^{1}$, different from (5.2). This other $S^{1}$ action on $U \times S^{1}$ can be described in the following way: let $\zeta=e^{2 \pi i t} \in S^{1}$. Then $\zeta$ acts on $U \times S^{1}$ by

$$
(u, s) \mapsto\left(u \rho^{k t}, s e^{2 \pi i k t}\right),
$$

where $\rho^{k t}=e^{2 \pi i h t}$ if $\rho=e^{2 \pi i h / k}$.

From this picture we see that if $k=1$ the action is free (i.e., the diffeomorphisms corresponding to $\zeta \neq 1$ have no fixed points), whereas when $k>1$ the action is free only in the complement of one orbit (that of the point $(0,1))$.

The following lemma is well known (see [28]).

Lemma 5.1. Let $S^{1}$ act smoothly, effectively, and without fixed point, on a compact orientable manifold of dimension 3 , and let $\Gamma$ be an orbit. Then $\Gamma$ has an invariant neighborhood $B$ such that the action on this neighborhood is diffeomorphic to the action of $S^{1}$ on $N$ described above, for a certain value of $k \geq 1$ 
and primitive $k$ th root of $1, \rho$. In fact, with the possible exception of finitely many orbits $\Gamma, k=\rho=1$.

We shall use this lemma to construct a complex surface $\bar{D}$ whose boundary is $M$. Cover $M$ with a finite collection $\mathscr{B}=\{B\}$ of invariant neighborhoods as in the lemma. If a $B \in \mathscr{B}$ is diffeomorphic to $N=N_{k, \rho}$, define a space $\bar{D}_{B}$ as the quotient of

$$
\widetilde{D}_{B}=U \times \bar{\Delta} \quad(\Delta=\text { unit disc })
$$

by the action of the group $\mathbb{Z} / k$ given by

$$
U \times \bar{\Delta} \ni(u, s) \mapsto\left(u \rho^{j}, s e^{2 \pi i j / k}\right) \in U \times \bar{\Delta}
$$

$j \in \mathbb{Z} / k$. For $j \not \equiv 0 \bmod k$ the diffeomorphism (5.4) has only one fixed point, $(0,0)$, so that the quotient $\bar{D}_{B}$ is manifold with boundary and one singularity (an orbifold). We shall endow $\bar{D}_{B}$ with the structure of a complex analytic space, glue these $\bar{D}_{B}$ 's together to form a compact analytic space $\bar{D}_{0}$ with boundary and isolated singularities; finally $\bar{D}$ will be obtained by desingularizing $\bar{D}_{0}$.

Step 1. Complex structure on $\widetilde{D}_{B}$. Let $\pi_{B}: \widetilde{D}_{B} \rightarrow \bar{D}_{B}$ denote the canonical projection, which is a smooth covering ramified at $(0,0)$. It restricts to a smooth covering

$$
\pi: U \times S^{1} \rightarrow N \approx B
$$

On $\widetilde{D}_{B}$ we have an obvious $S^{1}$-action

$$
(u, s) \mapsto(u, \zeta s),
$$

$\zeta \in S^{1}$. In fact, this formula defines an action $\left\{h_{\zeta}: \zeta \in \bar{\Delta}\right\}$ of the semigroup $\bar{\Delta}$ by letting $\zeta \in \bar{\Delta} .\left\{h_{\zeta}\right\}$ factors through $\pi_{B}$ to define a $\bar{\Delta}$-action on $\bar{D}_{B}$; then $\pi_{B}$ becomes $\bar{\Delta}$-equivariant. Similarly, in $(5.5)$ both $\pi$ and $\approx$ are $S^{1}$-equivariant.

Using (5.5) we can pull back the contact and CR structures from $B \subset M$ first to $N$, then to $U \times S^{1}$. Also, the Beltrami differentials $\mu(\zeta)$ on $B$ pull back to Beltrami differentials $\lambda(\zeta)$ on $U \times S^{1}$. If $\mu$ is a Beltrami differential along a nonexceptional trajectory $\Gamma_{1}$ then a Beltrami differential corresponding to any of the $k$ preimages of $\Gamma_{1}$ is simply $\lambda(\zeta)=\mu(\zeta)$. On the other hand, if $\mu$ corresponds to the exceptional trajectory $\Gamma$ then a Beltrami differential corresponding to the (only) preimage of $\Gamma$ is $\lambda(\zeta)=\mu\left(\zeta^{k}\right)$. In all cases we conclude that $\lambda$ extends holomorphically to $\Delta$ (and we shall denote this extension by the same letter $\lambda$ ).

The complex structure on $\widetilde{D}_{B}$ will be defined by the data of two independent $(0,1)$ vector fields, $\bar{Z}$ and $\bar{W} \cdot \bar{W}$ is easier to define:

$$
\bar{W}=\bar{s} \frac{\partial}{\partial \bar{s}},
$$

in coordinates $(u, s)$ on $\widetilde{D}_{B}=U \times \bar{\Delta}$. This is a $\bar{\Delta}$-invariant field. We record here that no matter what $\bar{Z}$ is, the choice of the above $\bar{W}$ as $(0,1)$ field makes 
the orbits of the $\bar{\Delta}$-action complex submanifolds in $\widetilde{D}_{B}$. Indeed, the mapping

$$
\Delta \ni s \mapsto(\text { const }, s) \in \widetilde{D}_{B}
$$

is holomorphic.

To get $\bar{Z}$, choose a transverse slice to the $S^{1}$-action $\left\{h_{\zeta}: \zeta \in S^{1}\right\}$ on $U \times S^{1}$, i.e., a surface $\Sigma$ that intersects every orbit at most once and then transversely. For example $\Sigma=U \times\{1\}$ will do: it intersects every orbit. Next construct a smooth section $X_{\Sigma}$ of $\left.\mathbb{C} \otimes H\left(U \times S^{1}\right)\right|_{\Sigma}$ consisting of $(1,0)$-like vectors (see $\S 3$ ), and extend it to a $\bar{\Delta}$-invariant vector field $X$ on the orbit of $\Sigma$. Any point $p \in \Sigma$ and the $(1,0)$-like vector $X_{p}$ determine a Beltrami differential $\lambda_{p}(\zeta)=$ $\lambda(p, \zeta)$, which extends to a smooth function of $(p, \zeta) \in \Sigma \times \bar{\Delta}$, holomorphically depending on $\zeta \in \Delta$. Furthermore

$$
|\lambda(p, \zeta)|<1, \quad(p, \zeta) \in \Sigma \times \bar{\Delta} .
$$

Now define the vector field $\bar{Z}$ on the $\bar{\Delta}$-orbit of $\Sigma$ by

$$
\bar{Z}_{h_{\zeta}(p)}=\bar{X}_{h_{\zeta}(p)}+\lambda(p, \zeta) X_{h_{\zeta}(p)}, \quad(p, \zeta) \in \Sigma \times \bar{\Delta} .
$$

The fields $\bar{Z}$ and $Z$ are independent because of (5.7), and transverse to the orbits of the $\bar{\Delta}$-action. The fields $\bar{W} / \bar{s}$ and $W / s$ are obviously independent and tangential to the orbits. Hence by declaring $\bar{Z}$ and $\bar{W}$ (or $\bar{W} / \bar{s}$ ) to be $(0,1)$ fields, we define a smooth almost complex structure on the $\bar{\Delta}$-orbit of $\Sigma$. To see that this structure is integrable, observe that $\operatorname{Re} \bar{W}$ and $\operatorname{Im} \bar{W}$ are infinitesimal generators of the $\bar{\Delta}$ action, and $X, \bar{X}$ are $\bar{\Delta}$-invariant, so that

$$
[\bar{W}, X]=[\bar{W}, \bar{X}]=0 \text {. }
$$

Hence

$$
[\bar{W}, \bar{Z}]=[\bar{W}, \bar{X}+\lambda X]=(\bar{W} \lambda) X=0,
$$

because $\lambda(p, \zeta)$ is holomorphic in $\zeta$, whence

$$
\bar{W}_{h_{\zeta}(p)} \lambda(p, \zeta)=\bar{\zeta} \frac{\partial}{\partial \bar{\zeta}} \lambda(p, \zeta)=0
$$

Thus by the Newlander-Nirenberg theorem we defined a complex structure on the orbit of $\Sigma$. If, for example $\Sigma=U \times\{1\}$, we get a complex structure on the whole of $\widetilde{D}_{B}$. It can be easily checked that the complex structure thus obtained does not depend on the choice of the slice $\Sigma$ and the vector field $X_{\Sigma}$. Indeed, it is only the invariant field $X$ that matters in the above construction; and if one replaces $X$ by another invariant field $X^{\prime}$ calculations as in the proof of Proposition 3.1 show that the corresponding field $\bar{Z}^{\prime}$ will be a multiple of $\bar{Z}$.

This also shows that, although the $S^{1}$-equivariant diffeomorphism $N \approx B$ in (5.5) is not unique and the complex structure on $\widetilde{D}_{B}$ depends on the choice of this diffeomorphism, different choices of diffeomorphisms lead to biholomorphic complex structures on $\widetilde{D}_{B}$. More precisely, two complex structures on $\widetilde{D}_{B}$ corresponding to different $S^{1}$-equivariant diffeomorphisms $N \approx B$ are related by a $\bar{\Delta}$-equivariant biholomorphism. The restriction of this biholomorphism to 
$\partial \widetilde{D}_{B}=U \times S^{1}$ covers the $S^{1}$-equivariant diffeomorphism $N \rightarrow N$ that relates the two different identifications $N \approx B$.

Step 2. The boundary of $\widetilde{D}_{B}$. The boundary of $\widetilde{D}_{B}, U \times S^{1}$ inherits a CR structure from $\widetilde{D}_{B}$, and it also has a CR structure that arises from the covering (5.5). These two are in fact the same, since the vector field $\left.\bar{Z}\right|_{U \times S^{1}}$ is a $(1,0)$ field for both structures (by the very definition of the complex structure on $\widetilde{D}_{B}$ on the one hand, and by the definition of the Beltrami differential $\lambda_{p}(\zeta)$ on the other).

Step 3. The complex structure on $\bar{D}_{B}$. Let $\Gamma_{0}$ be an orbit of the $S^{1}$-action on $\partial \widetilde{D}_{B}$ and $\Gamma_{j}$ its image under the diffeomorphism (5.4), $j \in \mathbb{Z} / k$. Then $\pi\left(\Gamma_{0}\right)=\pi\left(\Gamma_{j}\right)$; hence the Beltrami differentials $\lambda_{0}, \lambda_{j}$ corresponding to these two orbits are the same. It follows that the action of $\mathbb{Z} / k$ on $\widetilde{D}_{B}$, defined by (5.4), is a holomorphic action. Hence, by a theorem of H. Cartan (see [8]), the interior of the quotient space $\bar{D}_{B}$ is a complex space with possibly one isolated singularity. Since $\pi_{B}$ is a smooth covering near $\partial \widetilde{D}_{B}$ it also follows that the complex structure of int $\bar{D}_{B}$ smoothly extends to the whole of $\bar{D}_{B}$. Because of Step 2 the boundary $N$ of $\bar{D}_{B}$ inherits a CR structure from $\bar{D}_{B}$ which is isomorphic with $B$. Indeed, $\approx$ in $(5.5)$ is an $S^{1}$-equivariant $C R$ diffeomorphism.

Step 4. Gluing the $\bar{D}_{B}$ 's together. Let us summarize what we have done up to now. For a $\left\{g_{\zeta}: \zeta \in S^{1}\right\}$ invariant open set $B \subset M$ as in Lemma 5.1 and an $S^{1}$-equivariant isomorphism $\varphi: N \approx B \approx$ we constructed a complex space $\bar{D}_{B}$ with boundary $N$. The CR structure of $N$ as the boundary of $\bar{D}_{B}$ is such that $\varphi$ becomes a CR diffeomorphism. Moreover $\bar{D}_{B}$ admits a $\bar{\Delta}$-action which restricts to the $S^{1}$-action on $N$.

Suppose now that $B^{\prime} \subset M$ is another open set as in Lemma 5.1, $\varphi^{\prime}: N^{\prime} \rightarrow B^{\prime}$ a corresponding equivariant isomorphism, $\bar{D}_{B^{\prime}}$ the associated complex space. Suppose that $B \cap B^{\prime}$ is nonempty but does not contain an exceptional orbit (i.e. on $B \cap B^{\prime}$ the action $\left\{g_{\zeta}: \zeta \in S^{1}\right\}$ is free). Then $\varphi^{-1} \circ \varphi^{\prime}$ defines an $S^{1}$-equivariant CR diffeomorphism of an open invariant subset $P^{\prime} \subset N^{\prime}$ to an open invariant subset $P \subset N$. Because $B \cap B^{\prime}$ contains no exceptional orbit of the action $\left\{g_{\zeta}\right\}$, the $\bar{\Delta}$-orbits of $P$ resp. $P^{\prime}$ in $\bar{D}_{B}$ resp. $\bar{D}_{B^{\prime}}$ avoid the singular points of $\bar{D}_{B}$ resp. $\bar{D}_{B^{\prime}}$ (if they exist at all). The construction of the complex structure of $\bar{D}_{B}$ in Steps 1 and 3 implies that $\varphi^{-1} \circ \varphi^{\prime}$ extends to a $\bar{\Delta}$-equivariant biholomorphism $\phi_{B^{\prime} B}$ between these two orbits.

Now take a covering $\mathscr{B}=\{B\}$ of $M$ by invariant open sets $B$ as in Lemma 5.1. Every $B \in \mathscr{B}$ contains at most one exceptional $\left\{g_{\zeta}\right\}$-orbit; conversely, it can be assumed that every exceptional orbit is contained in only one $B \in \mathscr{B}$. Choosing equivariant diffeomorphisms $\varphi: N \underset{\rightarrow}{\approx} B$ as above, we obtain the spaces $\bar{D}_{B}$. With the help of the $\bar{\Delta}$-equivariant biholomorphisms $\phi_{B^{\prime} B}$ we can now glue the spaces $\bar{D}_{B}$ together to get a compact complex space $\bar{D}_{0}$ with 
boundary and isolated singularities inside $D_{0}$. In fact, the space $\bar{D}_{0}$ admits a $\bar{\Delta}$-action, since each $\bar{D}_{B}$ admitted one and the gluing was equivariant. The singular points are fixed by this action. Off the singular points the action is smooth (but, in general, not holomorphic).

Obviously, the boundary $\partial \bar{D}_{0}$ is CR equivalent to $M$, so that we can identify $M$ with the boundary of $\bar{D}_{0}$. Then the action $\left\{g_{\zeta}: \zeta \in S^{1}\right\}$ becomes the restriction of the $\bar{\Delta}$-action on $\bar{D}_{0}$ to $M$. For this reason in what follows we shall denote the $\bar{\Delta}$-action on $\bar{D}_{0}$ by $\left\{g_{\zeta}: \zeta \in \bar{\Delta}\right\}$.

Resolving the singularities of $\bar{D}_{0}$ we obtain a complex surface $\bar{D}$ with boundary $M$. Theorem 4.1 will be proved if we can prove that $\bar{D}$ is strictly pseudoconvex. Since this is a local property of $M=\partial \bar{D}$, and near $M, \bar{D}$ and $\bar{D}_{0}$ agree, we shall study $\bar{D}_{0}$.

Define a function $v: \bar{D}_{0} \rightarrow[0,1]$ by

$$
v(p)=\inf \left\{r^{2}: 0 \leq r \leq 1 \text { and } p \in g_{r}\left(\bar{D}_{0}\right)\right\}
$$

Equivalently, $v(p)=|\zeta|^{2}$ if $p \in g_{\zeta}\left(\partial \bar{D}_{0}\right)$.

Proposition 5.2. $v$ is smooth off the singular points of $\bar{D}_{0}$. The set $\{v=0\} \subset \bar{D}_{0}$ is a compact complex subspace of $D_{0}$ without boundary, of dimension 1 . It agrees with the fixed set of the action $\left\{g_{\zeta}: \zeta \in \bar{\Delta}\right\}$. Outside this set $v$ is strictly plurisubharmonic.

Proof. The only global statement in this proposition is about the compactness of the zero set of $v$, which is obvious in view of the continuity of $v$ and the compactness of $\bar{D}_{0}$. As for the rest, we can work locally, on open subsets $\bar{D}_{B} \subset \bar{D}_{0}$, or even on ramified covers $\widetilde{D}_{B}$. Indeed, for each $B \in \mathscr{B}, v$ lifts to a function $v_{B}$ on $\widetilde{D}_{B}=U \times \bar{\Delta}$ :

$$
v_{B}(u, s)=|s|^{2}
$$

since the $\bar{\Delta}$-action on $\widetilde{D}_{B}$ is given by (5.6). This shows that $v$ is smooth on $\bar{D}_{B}$ off the eventual ramification point of $\pi_{B}$, i.e., off the singular points of $\bar{D}_{0}$. It also shows that $\left\{v_{B}=0\right\}$ (and hence $\{v=0\}$ ) is the fixed set of the $\bar{\Delta}$-action on $\widetilde{D}_{B}$ (resp. on $\bar{D}_{0}$ ).

Next we want to show that the two real dimensional surface $\left\{v_{B}=0\right\}=F$ is a complex curve. Consider a $\bar{\Delta}$-invariant vector field $X$ on $\widetilde{D}_{B}$ as in Step 1 above. The mapping $h_{0}: \widetilde{D}_{B} \rightarrow \widetilde{D}_{B}$ corresponding to $\zeta=0 \in \bar{\Delta}$ maps $\widetilde{D}_{B}$ to $F$; hence the invariance of the field $X$ implies that $X$ is tangent to $F$ and the same holds for $\bar{X}$ and so by $(5.8)$ for $\bar{Z}$ and $Z$. Therefore the tangent spaces $T_{p} F(p \in F)$ are all complex subspaces of $T_{p} \widetilde{D}_{B}$, which implies that $F$ is a complex submanifold of $\widetilde{D}_{B}$. (From this it follows that $\{v=0\}$ is a complex subspace by virtue of Remmert's theorem on proper images of analytic sets, since $\pi_{B}$ is proper.)

What remains to prove is the strict plurisubharmonicity of $v$, which means 
that for $B \in \mathscr{B}$ the hermitian form

$$
(P, Q) \mapsto\left\langle\partial \bar{\partial} v_{B}, P \wedge \bar{Q}\right\rangle, \quad P, Q \in T_{p}^{1,0}\left(\widetilde{D}_{B}\right)
$$

is positive definite when $p \notin F$. We have for smooth sections $P, Q$ of $T^{1,0}\left(\widetilde{D}_{B}\right)$

$$
\begin{aligned}
\left\langle\partial \bar{\partial} v_{B}, P \wedge \bar{Q}\right\rangle & =\left\langle d \bar{\partial} v_{B}, P \wedge \bar{Q}\right\rangle \\
& =P\left\langle\bar{\partial} v_{B}, \bar{Q}\right\rangle-\bar{Q}\left\langle\bar{\partial} v_{B}, P\right\rangle-\left\langle\bar{\partial} v_{B}[P, \bar{Q}]\right\rangle \\
& =P\left(\bar{Q} v_{B}\right)-\left\langle\bar{\partial} v_{B},[P, \bar{Q}]\right\rangle
\end{aligned}
$$

We shall compute the form $\partial \bar{\partial} v_{B}$ in the basis $Z, W$ defined in Step 1 above. First put $P=Q=W$; we obtain

$$
\left\langle\partial \bar{\partial} v_{B}, W \wedge \bar{W}\right\rangle=W\left(\bar{W} v_{B}\right)=\frac{\partial^{2}|s|^{2}}{\partial s \partial \bar{s}}=1 .
$$

Second, put $P=W, Q=Z$, and observe that $X, \bar{X}$ and therefore $Z, \bar{Z}$ are tangential to the level sets $\left\{v_{B}=\right.$ const $\}$. Also by (5.8) and (5.9)

$$
[W, \bar{Z}]=[W, \bar{X}+\lambda X]=(W \lambda) X,
$$

which again is tangent to $\left\{v_{B}=\right.$ const $\}$. It is, in fact, a combination of $Z$ and $\bar{Z}$. So $(5.11)$ yields

$$
\left\langle\partial \bar{\partial} v_{B}, W \wedge \bar{Z}\right\rangle=0-0=0 .
$$

Third, put $P=Q=Z$; then

$$
\left\langle\partial \bar{\partial} v_{B}, Z \wedge \bar{Z}\right\rangle=0-\left\langle\bar{\partial} v_{B},[Z, \bar{Z}]\right\rangle .
$$

If we work on $\partial \widetilde{D}_{B}$ then we can write $Z=Y-i J Y$ with $Y, J Y$ real tangent vector fields to $\partial \widetilde{D}_{B}$, so that

$$
[Z, \bar{Z}]=2 i[Y, J Y] \text {. }
$$

Denoting by $V$ the infinitesimal generator of the action $\left\{h_{\zeta}: \zeta \in S^{1}\right\}$ on $\partial \widetilde{D}_{B}$, the positivity of the action implies that

$$
[Y, J Y] \equiv c V \quad \bmod Z, \bar{Z}
$$

where $c<0$. Now

$$
\left\langle\bar{\partial} v_{B}, V\right\rangle=\frac{1}{2}\left\langle d v_{B}, V+i J V\right\rangle=\frac{i}{2}(J V) v_{B},
$$

with $(J V) v_{B}<0$ by virtue of the form (5.6) of the action $\left\{h_{\zeta}\right\}$, and (5.10). Putting (5.15), (5.16), and (5.17) together, we obtain that

$$
\left\langle\bar{\partial} v_{B},[Z, \bar{Z}]\right\rangle<0
$$

holds on $\partial \widetilde{D}_{B}$. To extend (5.18) to the whole of $\widetilde{D}_{B} \backslash F$, observe that by $(5.8)$

$$
[Z, \bar{Z}]=[X+\bar{\lambda} \bar{X}, \bar{X}+\lambda X] \equiv\left(1-|\lambda|^{2}\right)[X, \bar{X}] \bmod Z, \bar{Z}
$$

with $|\lambda|<1$; that $[X, \bar{X}]$ is $\left\{h_{\zeta}: \zeta \in \bar{\Delta}\right\}$ invariant; and that $v_{B} \circ h_{\zeta}=|\zeta|^{2} v_{B}$. Therefore $\left\langle\bar{\partial} v_{B},[X, \bar{X}]\right\rangle$ does not change sign on a $\bar{\Delta} \backslash\{0\}$-orbit; by $(5.18)$ and 
(5.19) this sign is negative on $\partial \widetilde{D}_{B}$, hence on all of $\widetilde{D}_{B} \backslash F$. Thus (5.18) holds on $\widetilde{D}_{B} \backslash F$, i.e.,

$$
\left\langle\partial \bar{\partial} v_{B}, Z \wedge \bar{Z}\right\rangle>0 \text { on } \widetilde{D}_{B} \backslash F .
$$

(5.12), (5.13), and (5.20) together imply that $v_{B}$ is strictly plurisubharmonic. Since $v=1$ on $M, v<1$ on $D_{0}$, and $d v \neq 0$ on $M$, the boundary of $\bar{D}_{0}$ (or $\bar{D}$ ) is strictly pseudoconvex, which finishes the proof of Theorem 4.1.

6. Some remarks on Theorem 4.1. As we saw, $\bar{D}_{0}$ contains a compact complex curve $\{v=0\}$. The maximum principle applied to the plurisubharmonic function $v$ implies that there is no other compact complex curve (without boundary) contained in $\bar{D}_{0}$. Hence by blowing down the connected curve $\{v=0\}$ in $\bar{D}_{0}$, we obtain a normal Stein space $\bar{D}^{*}$ with boundary and at most one singularity. $(\{v=0\}$ is connected since it is the image of the connected manifold $M$ under $g_{0}$.) Since a normal Stein space is uniquely determined by its boundary, we have the following

Theorem 6.1. Suppose a strictly pseudoconvex three-dimensional CR manifold $M$ is the boundary of a compact normal Stein space with at least two isolated singularities. Then $M$ does not admit any inner $S^{1}$-action.

Let us return to $M$ as in Theorem 4.1, and $\bar{D}_{0}, v$ as in its proof. Similarly as $\partial \bar{\partial} v$ was computed in $\S 5$, we can compute $\partial \bar{\partial} \log v$ (or $\partial \bar{\partial} \log v_{B}$ ) to get

$$
\begin{aligned}
& \left\langle\partial \bar{\partial} \log v_{B}, W \wedge \bar{W}\right\rangle=0, \\
& \left\langle\partial \bar{\partial} \log v_{B}, W \wedge \bar{Z}\right\rangle=0, \\
& \left\langle\partial \bar{\partial} \log v_{B}, Z \wedge \bar{Z}\right\rangle>0 .
\end{aligned}
$$

Hence we have

Theorem 6.2. On $\bar{D}_{0} \backslash\{v=0\}$ the function $u=\log v$ is smooth and plurisubharmonic. Furthermore rk $\partial \bar{\partial} u=1$; in particular $u$ satisfies the Monge-Ampere equation $(\partial \bar{\partial} u)^{2}=0$.

The behavior of $u$ at the set $v=0$ can best be analysed when the inner $S^{1}$-action on $M$ is free, so that $\bar{D}_{0}$ and the curve $\{v=0\}$ are nonsingular, and moreover, if after blowing down the exceptional curve in $D_{0}$ we obtain a nonsingular Stein space $\bar{D}^{*}$. As observed before, $\bar{D}^{*}$ is uniquely determined by $M$ and does not depend on the choice of the inner action $\left\{g_{\zeta}\right\}$. In this case we can introduce some smooth Riemann metric on $\bar{D}^{*}$, and measure distances using this metric. The function $u$ descends to a function $u^{*}$ on $\bar{D}^{*}$; if $0 \in D^{*}$ denotes the image of the exceptional divisor, we have

Theorem 6.3. The function $u^{*}$ is the Green function of $\bar{D}^{*}$ associated with the Monge-Ampere operator $(\partial \bar{\partial})^{2}$, with pole at 0 . That is,

$$
\begin{cases}u^{*} \text { is plurisubharmonic } & \text { on } D^{*}, \\ \left(\partial \bar{\partial} u^{*}\right)^{2}=0 & \text { on } D^{*} \backslash\{0\} \\ u^{*}=0 & \text { on } \partial D^{*} \\ u^{*}(z)=2 \log \operatorname{dist}(0, z)+O(1) & \text { as } z \rightarrow 0\end{cases}
$$


In fact, the second equation above can be strengthened to rk $\partial \bar{\partial} u^{*}=1$; and the kernel of $\partial \bar{\partial} u^{*}$ is tangent to the orbit of the $\bar{\Delta}$-action that $\bar{D}^{*}$ inherits from $\bar{D}_{0}$. From Step 1 in Chapter 5 we know that the orbits of the $\bar{\Delta}$-action on $\bar{D}_{0}$, and hence on $\bar{D}^{*}$, are biholomorphic to the unit disc $\bar{\Delta}$. Furthermore, under this biholomorphism the center of the unit disc corresponds to $0 \in D^{*}$, and the $\bar{\Delta}$-action on an orbit corresponds to the action

$$
\bar{\Delta} \ni s \mapsto \zeta s \in \bar{\Delta}, \quad \zeta \in \bar{\Delta}
$$

on the unit disc. Although the biholomorphism between the orbits and the unit disc is determined only up to rotation (multiplication by $\omega \in S^{1}$ ), the image of the action (6.2) on a $\bar{\Delta}$-orbit is uniquely determined by the orbit as a submanifold in $\bar{D}_{0}$. Theorem 6.3 shows that the orbits are uniquely determined by the function $u^{*}$ (they are integral submanifolds of Ker $\partial \bar{\partial} u$ ). On the other hand, the minimum principle of Bedford and Taylor (see [3]) implies that $u^{*}$ is uniquely determined by $0 \in D^{*}$ as a solution of (6.1) (see [23]). This reasoning proves the following theorem:

Theorem 6.4. Suppose that $M$ is the strictly pseudoconvex boundary of a compact, two-dimensional Stein manifold $\bar{D}^{*}$. Then any free inner $S^{1}$-action on $M$ determines a point $0 \in D^{*}$, and to different actions different points of $D^{*}$ correspond. It follows that free inner $S^{1}$-actions on $M$ are parametrized by at most four real parameters.

There is a companion to Theorem 4.1 concerning outer actions.

Theorem 6.5. Suppose $M$ admits an outer $S^{1}$-action. Then it is the boundary of a strictly pseudoconcave complex surface; i.e., there is a compact complex surface $\bar{E}$ with boundary $M$ such that for any $p \in M$ there is a strictly plurisubharmonic function $u$ on some neighborhood of $p$, positive on $E$, vanishing on $M$, but $d u \neq 0$ on $M$.

Proof. The proof is the same as that of Theorem 4.1 with the difference that the role of the semigroup $\bar{\Delta}$ is taken over by the semigroup $\bar{\Delta}^{\prime}=\mathbb{P}_{1} \backslash \Delta$ (whose zero element is $\infty)$. Thus, in the definition of $\widetilde{D}_{B}$ and later $\bar{\Delta}$ should be replaced by $\bar{\Delta}^{\prime}$. As a result we obtain complex surfaces $\bar{E}_{0}, \bar{E}$ on which $\bar{\Delta}^{\prime}$ acts.

The function $v$ will now be defined by

$$
v(p)=\sup \left\{r^{2}: 1 \leq r \text { and } p \in g_{r}\left(\bar{E}_{0}\right)\right\}
$$

or $v(p)=|\zeta|^{2}$ if $p \in g_{\zeta}\left(\partial E_{0}\right)$. Thus $v: \bar{E}_{0} \rightarrow[1, \infty] . v$ is smooth and strictly plurisubharmonic off the set $\{v=\infty\}$, which agrees with the fixed set of the $\bar{\Delta}^{\prime}$ action. Also, $\{v=\infty\}$ is a compact complex curve. Details are left to the reader.

7. On the existence of inner $S^{1}$ actions. We shall now turn our attention to strictly linearly convex hypersurfaces in $\mathbb{C}^{2}$ and prove that they admit inner $S^{1}$ actions (Theorem 4.3). The existence of inner actions can be read off from the results of Chapter III in [4]. Nevertheless, we shall give here a unified treatment 
for inner and outer actions, based on certain properties of the Kobayashi metric (which was the source of [4] as well).

Let $\bar{D}=D \cup M$ be a smooth, compact, four-dimensional manifold with interior $D$, boundary $M$. Assume that $\bar{D}$ is endowed with a smooth almost complex structure $J_{D}$ which is integrable on $D$, and which makes $M$ a strictly pseudoconvex CR manifold. (Then $\bar{D}$ is either strictly pseudoconvex or strictly pseudoconcave.)

Lemma 7.1. Suppose that for every $p \in M$ a smooth immersion $f=f^{p}: \bar{\Delta} \rightarrow \bar{D}$ and a smooth rank one subbundle $E=E^{p}$ of the pull back bundle $f^{*} T^{1,0} \bar{D}$ are defined so that

(a) $f$ and $E$ are holomorphic on $\Delta$;

(b) $f(1)=p, f\left(S^{1}\right) \subset M$;

(c) for every $\zeta \in \bar{\Delta}, f_{*}\left(T_{\zeta}^{1,0} \bar{\Delta}\right)$ and the fiber $E_{\zeta}$ are transverse;

(d) for $\zeta \in S^{1}, E_{\zeta}$ is tangent to $M$;

(e) if $P=f^{p}(\omega)$ for some $\omega \in S^{1}$ then

$$
f^{P}(\zeta)=f^{p}(\zeta \omega)
$$

(f) putting $F(p, \zeta)=f^{p}(\zeta), F: M \times \bar{\Delta} \rightarrow \bar{D}$ is smooth, for fixed $\zeta \in$ $\bar{\Delta} \backslash\{0\}, F(\cdot, \zeta): M \rightarrow \bar{D}$ is an immersion, and $F_{*}\left(T_{p} M \times\{0\}\right) \subset E_{0}^{p} \oplus$ $\bar{E}_{0}^{p}$.

Define an $S^{1}$ action $\left\{g_{\zeta}\right\}$ on $M$ by $g_{\zeta}(p)=f^{p}(\zeta)$. Then this action is a transverse contact action. If it is positive then it is inner; otherwise the reverse action $g_{\zeta^{-1}}$ is outer. If, in addition, the maps $f^{p}$ are embeddings, and the holomorphic discs $f^{p}(\bar{\Delta})$ foliate $\bar{D}$, then $\bar{D}$ is biholomorphic to the pseudoconvex surface constructed in the proof of Theorem 4.1 (denoted there $\bar{D}_{0}=\bar{D}$ ) or to the pseudoconcave surface constructed in the proof of Theorem 6.5 (denoted there $\left.\bar{E}_{0}=\bar{E}\right)$. Under the biholomorphism $M$ is fixed and $F(M \times\{0\})$ corresponds to the curve $\{v=0\}$, resp. $\{v=\infty\}$.

Observe that (e) implies that $\left\{g_{\zeta}\right\}$ is indeed a group action. Also, if the maps $f^{p}$ are embeddings, the action $\left\{g_{\zeta}\right\}$ is free, and so in the proof of Theorem 4.1 the space $\bar{D}_{0}$ has no singularities. As a result, in Step 4 of that proof no singularities are there to be resolved, so $\bar{D}=\bar{D}_{0}$ there. The same goes for the proof of Theorem 6.5.

Before we prove the lemma, we shall introduce a notation. There is an $(\mathbb{R}$ linear) isomorphism $\pi^{1,0}: T \bar{D} \rightarrow T^{1,0} \bar{D}$ given by

$$
\pi^{1,0} \xi=\left(\xi-i J_{D} \xi\right) / 2 \quad(\xi \in T \bar{D})
$$

(We shall also denote by $\pi^{1,0}$ the $\mathbb{C}$-linear extension: $\mathbb{C} \otimes T \bar{D} \rightarrow T^{1,0} \bar{D}$ of the above mapping.) If $\omega$ is a $(1,0)$ form on $\bar{D}$, then $\langle\omega, \xi\rangle=\left\langle\omega, \pi^{1,0} \xi\right\rangle$. We have the following simple proposition: 
Proposition 7.2. Suppose $h: \Delta \times(\varepsilon, \varepsilon) \rightarrow D$ is a continuously differentiable map, holomorphic in its first variable $\zeta \in \Delta$. Put

$$
\xi(\zeta)=\left.\frac{d}{d t}\right|_{t=0} h(\zeta, t)
$$

Then $\pi^{1,0} \xi$ is a holomorphic section of the pull back bundle $h_{0}^{*} T^{1,0} D$, where $h_{0}(\zeta)=h(\zeta, 0)$.

Proof. It suffices to prove that if $G$ is a holomorphic function locally defined in $D$ then $\left(\pi^{1,0} \xi\right) G$ is holomorphic. Now

$$
\begin{aligned}
\left(\pi^{1,0} \xi\right) G & =\left\langle d G, \pi^{1,0} \xi\right\rangle=\langle d G, \xi\rangle \\
& =\left.\frac{d}{d t}\right|_{t=0} G(h(\cdot, t)),
\end{aligned}
$$

which, as a locally uniform limit of holomorphic functions, is indeed holomorphic.

Proof of Lemma 7.1. First we want to prove that $\left\{g_{\zeta}\right\}$ is a transverse contact action. Let $p \in M$. With $f=f^{p}$ and $E=E^{p}$ as in the lemma, observe that $\pi^{1,0} H_{p} M=E_{1}^{p}$. According to (c) of the lemma, $E_{1}^{p}$ and $f_{*}\left(T_{1}^{1,0} \bar{\Delta}\right)$ are transverse; since $f_{*}\left(T_{1}^{1,0} \bar{\Delta}\right)$ is spanned by the vector

$$
\left.\pi^{1,0} \frac{d}{d \theta}\right|_{\theta=0} f\left(e^{i \theta}\right)=\left.\pi^{1,0} \frac{d}{d \theta}\right|_{\theta=0} g_{e^{i \theta}}(p),
$$

it follows that $\left(d g_{e^{i \theta}}(p) / d \theta\right)_{\theta=0}$ and $H_{p} M$ are transverse; i.e., the action $\left\{g_{\zeta}\right\}$ is transverse.

Now we shall define a smooth $(1,0)$ form $\sigma$ along $f$ (which means that for $\zeta \in \bar{\Delta}, \sigma(\zeta)$ is a $(1,0)$ form on $\left.\mathbb{C} \otimes T_{f(\zeta)}(\bar{D})\right)$ by

$$
\begin{aligned}
\left\langle\sigma, f_{*} \frac{\partial}{\partial \zeta}\right\rangle & =1, \\
\langle\sigma, v\rangle & =0 \quad \text { if } v \in E .
\end{aligned}
$$

Because of (c) this is a legitimate definition; and $\sigma$ is holomorphic over $\Delta$.

Take now a contact vector $Y \in H_{p} M$. Let $p(t)$ be smooth curve on $M$ with $(d p / d t)_{t=0}=Y$, and put $\xi(\zeta)=\left(d f^{p(t)}(\zeta) / d t\right)_{t=0}=F_{*}(\cdot, \zeta) Y$. Then $\xi(1)=Y$, and, more generally

$$
g_{\zeta *} Y=\xi(\zeta) \quad\left(\zeta \in S^{1}\right)
$$

We need to prove $\xi(\zeta) \in H M$.

In view of Proposition 7.2

$$
\langle\sigma, \xi\rangle=\left\langle\sigma, \pi^{1,0} \xi\right\rangle=\varphi
$$

is a holomorphic function on $\Delta$. Because of assumption (f), $\xi(0) \in E_{0} \oplus \bar{E}_{0}$; i.e., $\pi^{1,0} \xi(0) \in E_{0}$, so that $\varphi(0)=0$. 
For $\zeta \in S^{1}, \xi(\zeta)$ certainly is a tangent vector to $M$. Because $\operatorname{Im} \zeta \frac{\partial}{\partial \zeta}$ is tangent to $S^{1}, f_{*} \operatorname{Im} \zeta \frac{\partial}{\partial \zeta}$ is also tangent to $M$. This latter vector being transverse to $H M$, we can write

$$
\xi(\zeta)=V+a f_{*} \operatorname{Im} \zeta \frac{\partial}{\partial \zeta}
$$

with $V \in H M$, and $a \in \mathbb{R}$ ( $a$ of course may depend on $\zeta$ ). Hence for $\zeta \in S^{1}$

$$
\begin{aligned}
\varphi(\zeta) / \zeta & =\langle\sigma(\zeta), \xi(\zeta)\rangle / \zeta \\
& =\left\langle\sigma(\zeta), \pi^{1,0} V+a \pi^{1,0} f_{*} \operatorname{Im} \zeta \frac{\partial}{\partial \zeta}\right\rangle / \zeta \\
& =-a\left\langle\sigma(\zeta), i f_{*} \zeta \frac{\partial}{\partial \zeta}\right\rangle /(2 \zeta)=-i a / 2,
\end{aligned}
$$

by (7.1). Thus the harmonic function $\operatorname{Re} \varphi(\zeta) / \zeta$ is 0 when $\zeta \in S^{1}$, so it is identically zero on $\bar{\Delta}$, and the holomorphic function $\varphi(\zeta) / \zeta$ is constant. This constant has to be 0 , because $\pi^{1,0} Y \in E_{1}$, so that

$$
\varphi(1)=\langle\sigma(1), Y\rangle=\left\langle\sigma(1), \pi^{1,0} Y\right\rangle=0 .
$$

Thus $\varphi \equiv 0$. (7.4) now implies $a=0$, and by (7.3) $\xi(\zeta) \in H M$. Taking (7.2) into account, it follows that $g_{\zeta}$ is a contact diffeomorphism.

Next we want to prove that $\left\{g_{\zeta}\right\}$ is inner or $\left\{g_{\zeta^{-1}}\right\}$ is outer. For this, we shall study Beltrami differentials associated with trajectories. Let $0 \neq X_{p} \in$ $\mathbb{C} \otimes H_{p} M$ be a $(1,0)$-like vector. Then the vectors $\operatorname{Re} X_{p}=Y, \quad \operatorname{Im} X_{p}=Y^{\prime}$ are independent. Associate with $Y \in H_{p} M$ the vector field $\xi$ (along $f=f^{p}$ ) as in the first half of this proof; let $\eta$ be the corresponding field associated with $Y^{\prime} \in H_{p} M$. Thus $\xi, \eta$ are sections of $f^{*} T \bar{D}$; for $\zeta \in S^{1}$ (cf. (7.2))

$$
g_{\zeta *} Y=\xi(\zeta) \in H_{g_{\zeta}(p)} M, \quad g_{\zeta *} Y^{\prime}=\eta(\zeta) \in H_{g_{\zeta}(p)} M
$$

whence

$$
g_{\zeta *} X_{p}=\xi(\zeta)+i \eta(\zeta)
$$

The Beltrami differential $\mu(\zeta)$ determined by $X$ has the property that

$$
\xi(\zeta)-i \eta(\zeta)+\mu(\zeta)(\xi(\zeta)+i \eta(\zeta))
$$

is a $(0,1)$ vector for $\zeta \in S^{1}$. Applying $\pi^{1,0}$ to this vector we get 0 :

$$
\left(\pi^{1,0} \xi-i \pi^{1,0} \eta\right)+\left(\pi^{1,0} \xi+i \pi^{1,0} \eta\right) \mu=0
$$

where, for brevity, we dropped the $\zeta$ 's. Since $\pi^{1,0} \xi, \pi^{1,0} \eta$ are not identically vanishing holomorphic sections, (7.5) defines a meromorphic continuation of $\mu$ to $\Delta$. We shall use the same letter $\mu$ to denote this continuation.

For no $\zeta \in \Delta \backslash\{0\}$ can $|\mu(\zeta)|$ be 1 . Indeed, writing (7.5) in the form

$$
\pi^{1,0} \xi=i \frac{1-\mu}{1+\mu} \pi^{1,0} \eta
$$


we see that $|\mu(\zeta)|=1$ implies that $\pi^{1,0} \xi(\zeta)$ and $\pi^{1,0} \eta(\zeta)$ are dependent over the reals, hence so are $\xi(\zeta)=F_{*}(\cdot, \zeta) Y$ and $\eta(\zeta)=F_{*}(\cdot, \zeta) Y^{\prime}$. This, however, contradicts the assumption that $F(\cdot, \zeta)$ is an immersion.

Since $|\mu(\zeta)|<1$ for $\zeta \in S^{1}$, and $|\mu(\zeta)| \neq 1$, for $\zeta \in \Delta$ minus a finite set, it follows that $|\mu(\zeta)|<1$ throughout $\Delta$ with the possible exception of finitely many points $\zeta$ (poles of $\mu$, and 0 ). Bounded singularities being removabale, we can conclude that $\mu$ has no poles at all, and so the Beltrami differentials extend holomorphically to $\Delta$.

Hence, if the action $\left\{g_{\zeta}\right\}$ is positive, it is inner. On the other hand, if the reverse action $\left\{g_{\zeta^{-1}}\right\}$ is positive then the Beltrami differentials associated with this action are $\lambda(\zeta)=\mu\left(\zeta^{-1}\right)$ rather than $\mu(\zeta)$, and $\lambda$ obviously has a holomorphic extension to $\mathbb{P}_{1} \backslash \Delta$.

When the $f^{p}$ 's are embeddings and $f^{p}(\bar{\Delta})$ foliate $\bar{D}$, the circle action $\left\{g_{\zeta}\right\}$ extends to a disc action on $\bar{D}$ (also denoted $g_{\zeta}$ ):

$$
\bar{D} \ni Z=f^{p}(\omega) \mapsto f^{p}(\zeta \omega) \quad(\zeta \in \bar{\Delta}),
$$

and there is an obvious $\bar{\Delta}$-equivariant diffeomorphism $\delta$ from our $\bar{D}$ to $\bar{D}_{0}$ of the proof of Theorem 4.1, which is the identity on $M$. This diffeomorphism is holomorphic along the orbits of the $\bar{\Delta}$ action. To see holomorphicity in transverse directions, observe that because (7.5) holds for $\zeta \in \bar{\Delta}$

$$
g_{\zeta *} \bar{X}_{p}+\mu(\zeta) g_{\zeta *} X_{p} \in T^{0,1} \bar{D} \quad(\zeta \in \bar{\Delta})
$$

A comparison with (5.8) now yields that the diffeomorphism $\delta$ is biholomorphic, and the proof of Lemma 7.1 complete.

Remark 7.3. Since for $\zeta \in S^{1} E_{\zeta}^{p}=\pi^{1,0} H_{f^{p}(\zeta)} M$, it follows that $E^{p}$ is uniquely determined by $p$, and also that $E_{\zeta}^{p}$ depends smoothly on $(p, \zeta) \in M \times \bar{\Delta}$. Furthermore, if $P=f^{p}(\omega)$ with $\omega \in S^{1}$ then $E_{\zeta}^{P}=E_{\zeta \omega}^{p}$.

Proof of Theorem 4.3, part 1. We shall apply Lemma 7.1 to produce an inner action on $M$. Let $M=\partial D$ with $D \subset \mathbb{C}^{2}$ strictly linearly convex, and fix a point $0 \in D$. For $v \in T_{0}^{1,0} D, v \neq 0$, consider Royden's variational problem

$$
\max \left\{\lambda>0 \mid \exists f: \Delta \rightarrow D \text { holomorphic such that } f_{*}(0) \frac{\partial}{\partial \zeta}=\lambda v\right\} .
$$

The maximum defines the infinitesimal form of the Kobayashi metric (see $[15,33])$. Here we shall rather be concerned with the maximizing functions in (7.6), which are called extremal mappings. In all this proof 0 will be kept fixed, but $v$ will be let to vary, so that extremal mappings $f$ will always map $0 \in \Delta$ to $0 \in D$ but in general $f_{*}(0) \frac{\partial}{\partial \zeta}$ will be different for different extremal maps $f$.

It is known (see [21] for the convex and [22] for the linearly convex case) that extremal maps extend smoothly to $\bar{\Delta}$ and map $S^{1}$ into $M=\partial D$. Furthermore, given $p \in M$ there is a unique extremal map $f=f^{p}$ such that $f(1)=p$. Also, 
for any extremal mapping $f: \bar{\Delta} \rightarrow \bar{D}$ there is a smooth $(1,0)$ form $\sigma$ along $f$, holomorphic on $\Delta$ such that

$$
\langle\sigma(\zeta), Y\rangle=0 \quad \text { if } \zeta \in S^{1}, Y \in H_{f(\zeta)} M
$$

and

$$
\left\langle\sigma(\zeta), f_{*} \frac{\partial}{\partial \zeta}\right\rangle=1 \text { if } \zeta \in \bar{\Delta}
$$

The form $\sigma$ does not explicitly occur in $[21,22]$, but is related to the mapping $\widetilde{f}=\left(\widetilde{f}_{1}, \widetilde{f}_{2}\right): \bar{\Delta} \rightarrow \mathbb{C}^{2}$ appearing there by $\sigma=\widetilde{f}_{1} d z_{1}+\widetilde{f}_{2} d z_{2}, z_{1}, z_{2}$ being the coordinates on $\mathbb{C}^{2}$, and $(7.7),(7.8)$ are proved in terms of $\widetilde{f}_{1}, \widetilde{f}_{2}$.

Now define a line bundle $E=E^{p} \subset f^{*} T^{1,0} \bar{D}$ as the kernel of $\sigma$. In this way we have associated with every $p \in M$ and $f^{p}$ and $E^{p}$ as in Lemma 7.1. Let us check that (a) through (f) of this lemma are satisfied.

(a) and (b) are basic properties of extremal mappings as explained above. (c) and (d) follow from (7.8) and (7.7), respectively. (e) follows from the simple fact that if $f(\zeta)$ is extremal and $\omega \in S^{1}$ then $f(\omega \zeta)$ is extremal, too. As to (f), that $F(p, \zeta)=f^{p}(\zeta)$ is smooth was proved in [21,22]. Also, with the "spherical representation" $\Phi$ of $\bar{D}$ on the ball, as in [21, Théorème 3] we can write

$$
F(p, \zeta)=\Phi^{-1}(\zeta \Phi(p))
$$

Since in [22] it is proved that $\Phi$ is a smooth diffeomorphism off 0 , for any $\zeta \in \bar{\Delta}, \zeta \neq 0, F(\cdot, \zeta)$ is actually a smooth embedding. In fact, $F$ maps $M \times$ $(0,1]$ diffeomorphically to $\bar{D} \backslash\{0\}$. (The corresponding theorem for linearly convex domains is not formulated in [22] but follows directly from the smooth dependence of extremals on parameters (see Theorem 5) there.)

The last condition in (f) is trivially satisfied in view of $F(p, 0)=0$, for this implies $F_{*}\left(T_{p} M \times\{0\}\right)=\{0\}$.

Thus the assumptions of Lemma 7.1 are satisfied. We claim that the action $\left\{g_{\zeta}\right\}$ constructed there is positive.

Indeed, let $v$ be a strictly plurisubharmonic defining function of $D$, and $Y$ a section of $H M$ near a point $p \in M$. Then, using (5.11)

$$
0<\langle\partial \bar{\partial} v,(Y-i J Y) \wedge(Y+i J Y)\rangle=2 i\langle\bar{\partial} v,[J Y, Y]\rangle .
$$

On the other hand, with the extremal mapping $f=f^{p}$, the infinitesimal generator of the action $\left\{g_{\zeta}\right\}$ in the point $p$ is $-2 f_{*} \operatorname{Im} \zeta \frac{\partial}{\partial \zeta}$. Now

$$
\begin{aligned}
2 i\left\langle\bar{\partial} v,-2 f_{*} \operatorname{Im} \zeta \frac{\partial}{\partial \zeta}\right\rangle & =2\left\langle\bar{\partial} v, f_{*} \bar{\zeta} \frac{\partial}{\partial \bar{\zeta}}\right\rangle \\
& =2\left\langle v \circ f, \bar{\zeta} \frac{\partial}{\partial \bar{\zeta}}\right\rangle>0 .
\end{aligned}
$$

This latter inequality (valid for $|\zeta|=1$ ) can be checked by writing $v(f(\zeta))=$ $\left(|\zeta|^{2}-1\right) \rho(\zeta)$ with some positive smooth function $\rho$. Since $\bar{\partial} v$ vanishes on $H M,(7.10)$ and (7.11) show that the action $\left\{g_{\zeta}\right\}$ is indeed positive. Hence by 
Lemma 7.1, it is inner, and so we proved the part of Theorem 4.5 about inner actions.

Before proceeding to outer actions, observe that the $S^{1}$ action constructed above is free, since extremal maps $f$ embed $\bar{\Delta}$ into $\bar{D}$. From the argument leading to Theorem 6.4 it easily follows that letting the base point 0 vary in $D$, the above construction yields all free inner $S^{1}$ actions on $M$.

8. Outer actions. In what follows we shall need the following.

Proposition 8.1. If $D \subset \mathbb{C}^{2}$ is a strictly linearly convex domain with smooth boundary $M$ and $q \in \mathbb{C}^{2} \backslash \bar{D}$ then there is a (complex, affine) line through $q$ that does not intersect $\bar{D}$.

Proof. First observe that if $L$ is a line such that $L \cap D \neq \varnothing$ then $L$ cannot be tangent to $M$ by linear convexity, and hence $L$ intersects $M$ transversely. Therefore if $L$ is continuously varied so that $L \cap D \neq \varnothing$ then the topological type of the smooth curve $L \cap M$ does not change. Since $L$ can be moved to a position where $L \cap M$ is homeomorphic to the circle (say, when $L$ is near an extremal point of $M$ ), it follows that for every $L, L \cap D$ is either homeomorphic to a disc or empty.

As a second step adjoin a $\mathbb{P}_{1}$ to $\mathbb{C}^{2}$ to get projective space $\mathbb{P}_{2}$. We want to show that there is a (projective) line $\ell \subset \mathbb{P}_{2}$ through $q$ that does not intersect $D$. If this were not true then for every line $\ell \subset \mathbb{P}_{2}$ through $q, \ell \cap M$ would be a smooth Jordan curve varying smoothly with $\ell$, and $\ell \cap\left(\mathbb{P}_{2} \backslash D\right)$ would be a simply connected domain with smooth boundary, again varying smoothly with $\ell$. These simply connected domains could be contracted to $q \in \ell \cap\left(\mathbb{P}_{2} \backslash D\right)$, and if these contractions are performed with a little care, they define a contraction of the whole of $\mathbb{P}_{2} \backslash D$ on $q$. Thus $\mathbb{P}_{2} \backslash D$ would be contractible, and its Euler characteristic $\chi\left(\mathbb{P}_{2} \backslash D\right)=1$.

Similarly, using an interior point $Q \in D$ instead of $q$, we get that also $\bar{D}$ is contractible. Observe that $\chi(M)=0$, because $M$ is an odd-dimensional closed manifold. These values, however, contradict

$$
3=\chi\left(\mathbb{P}_{2}\right)=\chi(\bar{D})+\chi\left(\mathbb{P}_{2} \backslash D\right)-\chi(M)
$$

(For the above topological facts see, e.g., [34]). The contradiction shows that there is a projective line $\ell$, hence also an affine line $L$, through $q$ that is disjoint from $D$.

If $L$ is disjoint from $M$, too, then we are done. Otherwise it touches $M$ in a single point $p$. Denote by $v$ an outward normal vector to $M$ in $p$. It is then easy to check that for small positive $\varepsilon$ the line $L_{\varepsilon}$ passing through $q$ and $p+\varepsilon v$ does not intersect $\bar{D}$, and the proof is complete.

We shall also need the notion of the dual of a strictly linearly convex domain $D$. As before, embed $\mathbb{C}^{2}$ into projective space $\mathbb{P}_{2}=\mathbb{C}^{2} \cup \mathbb{P}_{1}$, and in the dual projective space $\mathbb{P}_{2}^{*}$ consisting of (complex, projective) lines in $\mathbb{P}_{2}$ consider the dual $D^{\prime}$ of $D$. It is defined as the set of those lines $q^{\prime} \subset \mathbb{P}_{2}$ that do not intersect the closure of $D . D^{\prime}$ is a domain whose boundary $M^{\prime}$ consists of lines in $\mathbb{P}_{2}$ that are tangent to $M=\partial D$. 
Proposition 8.2. For $D$ a strictly linearly convex, smooth domain, $D^{\prime}$ is also smoothly bounded and strictly linearly convex.

Proof. The mapping $\gamma$ which sends a point $q \in M=\partial D$ to the line $q^{\prime}$ tangent to $M$ in $q$ is clearly smooth and one to one onto $M^{\prime}$. To see that $M^{\prime}$ is a smooth hypersurface we shall construct a smooth left inverse of $\gamma$ as follows. Let $r$ be a smooth defining function of $D$. Then for $q \in M, \gamma(q)=q^{\prime}$ implies that the restriction of $r$ to the line $q^{\prime}$ assumes its minimum in $q$. Observe that strict linear convexity implies that this minimum is nondegenerate. Thus if $q^{\prime}$ varies now in a small neighborhood of $M^{\prime}$, there will be a unique point $\gamma^{\prime}\left(q^{\prime}\right) \in q^{\prime} \subset \mathbb{P}_{2}$ where the restriction $\left.r\right|_{q^{\prime}}$ assumes its minimum. Furthermore, this minimum point depends smoothly on $q^{\prime}$. Since $\gamma^{\prime} \circ \gamma=\mathrm{id}_{M}, M^{\prime}$ is indeed smooth.

As to strict linear convexity of $D^{\prime}$, see Lemma 5.2 in [24].

The dual of $\mathbb{P}_{2}^{*}$ can be identified with $\mathbb{P}_{2}:$ if $\ell \subset \mathbb{P}_{2}^{*}$ is a line, then points $q^{\prime} \in \ell$ correspond to a pencil of lines in $\mathbb{P}_{2}$ passing through a point $q$. The identification is

$$
\mathbb{P}_{2}^{* *} \ni \ell \leftrightarrow q \in \mathbb{P}_{2} .
$$

From Proposition 8.1 it is easy to deduce that under this identification, the dual of $D^{\prime}$ becomes $D$, and so $M^{\prime \prime}=M$.

We shall need one more construction before embarking on the proof of the second part of Theorem 4.3. Let $q^{\prime} \in \mathbb{P}_{2}^{*}$ and $L$ be a one-(complex) dimensional subspace in $T_{q}^{1,0} \mathbb{P}_{2}^{*}$. Then there is a unique line $\ell \subset \mathbb{P}_{2}^{*}$ through $q^{\prime}$ such that $T_{q^{\prime}}^{1,0} \ell=L$. This line $\ell$ corresponds to a point $q \in \mathbb{P}_{2}$ under the identification (8.1); put $q=\rho(L)$.

A "dual" operation to $\rho$ is the following. Let $q^{\prime}$ be a line in $\mathbb{P}_{2}, q \in q^{\prime}$. Denote the line $T_{q}^{1,0} q^{\prime} \subset T_{q}^{1,0} \mathbb{P}_{2}$ by $\varepsilon\left(q, q^{\prime}\right) . \varepsilon$ is, in some sense, the dual of $\rho$.

It is easy to check (using local coordinates for example) that if $q^{\prime}(\zeta)$ and $L_{\zeta} \subset T_{q^{\prime}(\zeta)}^{1,0} \mathbb{P}_{2}^{*}$ depend holomorphically on $\zeta \in \Delta$ then $\rho\left(L_{\zeta}\right)$ is a holomorphic mapping of $\Delta$ into $\mathbb{P}_{2}$. Similarly, if $q(\zeta)$ and $q^{\prime}(\zeta)$ are holomorphic, $q(\zeta) \in$ $q^{\prime}(\zeta)$, then $\varepsilon\left(q(\zeta), q^{\prime}(\zeta)\right) \subset T_{q(\zeta)}^{1,0} \mathbb{P}_{2}$ depends holomorphically on $\zeta$, and thus defines a holomorphic line bundle over $\Delta$.

Proof of Theorem 4.3, part 2. The construction of outer actions is based on the observation that the diffeomorphism $\gamma: M \rightarrow M^{\prime}$ defined in the proof of Proposition 8.2 sets up a correspondence between inner actions on $M$ (resp. $M^{\prime}$ ) and certain outer actions on $M^{\prime}$ (resp. $M$ ). More precisely, to define mappings $f^{p}$ and bundles $E^{p}$ as in Lemma 7.1 for $M$, we shall use the corresponding objects $f^{p^{\prime}}, E^{p^{\prime}}$ for $M^{\prime}$.

Observe that the line at infinity in $\mathbb{P}_{2}$ does not intersect $\bar{D}$; thus the corresponding point $0^{\prime} \in \mathbb{P}_{2}^{*}$ is in $D^{\prime}$. Also, if we choose a point $0 \in D$ (which we can assume to be the origin in $\mathbb{C}^{2}$ ), then any line $q^{\prime}$ through 0 intersects $D$ and hence the corresponding line in $\mathbb{P}_{2}^{*}$-and also lines close to it-will be 
disjoint from $D^{\prime}$. Thus, if the line corresponding to 0 is removed from $\mathbb{P}_{2}^{*}, D^{\prime}$ will become a bounded (strictly linearly convex) domain in the remaining affine space, which can be identified with $\left(\mathbb{C}^{2}\right)^{*}$.

Construct extremal mappings $f^{p^{\prime}}: \bar{\Delta} \rightarrow \bar{D}^{\prime}$ and associated line bundles $E^{p^{\prime}} \subset f^{p^{*} *} T^{1,0} \bar{D}^{\prime}$ as in the first part of this proof, in $\S 7 ; p^{\prime} \in M^{\prime}, f^{p^{\prime}}(0)=0^{\prime}$. With the help of these objects we shall construct mappings $f^{p}: \bar{\Delta} \rightarrow \mathbb{P}_{2} \backslash D$ and bundles $E^{p}(p \in M)$ that satisfy the conditions of Lemma 7.1 (with $\bar{D}$ replaced by $\left.\mathbb{P}_{2} \backslash D\right)$ as follows.

Let $p \in M$ and $\zeta \in \bar{\Delta}$. Denote by $p^{\prime}=\gamma(p)$ the line in $\mathbb{P}_{2}$ that is tangent to $M$ in $p$. Then $p^{\prime} \in M^{\prime}$. With $\rho$ and $\varepsilon$ defined above, put

$$
f^{p}(\zeta)=\rho\left(E_{\zeta}^{p^{\prime}}\right), \quad E_{\zeta}^{p}=\varepsilon\left(f^{p}(\zeta), f^{p^{\prime}}(\zeta)\right) .
$$

Since $E_{\zeta}^{p^{\prime}} \subset T_{q^{\prime}}^{1,0} \mathbb{P}_{2}$ with $q^{\prime}=f^{p^{\prime}}(\zeta), f^{p}(\zeta) \in f^{p^{\prime}}(\zeta)$, so that the second part of (8.2) makes sense. $f^{p}: \bar{\Delta} \rightarrow \mathbb{P}_{2}$ is a smooth mapping and $E^{p}=\left\{E_{\zeta}^{p}\right\}$ is a smooth subbundle of $f^{*} T^{1,0} \mathbb{P}_{2}$; both $f^{p}$ and $E^{p}$ are holomorphic on $\Delta$, and depend smoothly on $p \in M$.

Actually $f^{p}$ maps into $\mathbb{P}_{2} \backslash D$. Indeed, if for some $\zeta \in \bar{\Delta}, \ell \subset \mathbb{P}_{2}^{*}$ is the line determined by

$$
T_{f^{p^{\prime}(\zeta)}}^{1,0} \ell=E_{\zeta}^{p^{\prime}}
$$

then, of course, $\ell$ intersects $\bar{D}^{\prime}\left(f^{p^{\prime}}(\zeta) \in \ell \cap D^{\prime}\right)$, whence $\ell \in \mathbb{P}_{2}^{* *} \backslash D^{\prime \prime}$. After identification (8.1) this becomes $f^{p}(\zeta)=\rho\left(E_{\zeta}^{p^{\prime}}\right) \in \mathbb{P}_{2} \backslash D$.

To apply Lemma 7.1 we have to check that $f=f^{p}$ and $E=E^{p}$ satisfy the assumptions there. Some verifications are immediate. We have already mentioned that (a) holds. As to (b), let $\zeta \in S^{1}$. Then $E_{\zeta}^{p^{\prime}}=H_{f^{p^{\prime}}(\zeta)}^{1,0} M^{\prime}$, so that $f^{p}(\zeta)=\rho\left(E_{\zeta}^{p^{\prime}}\right)$ is the line through $f^{p^{\prime}}(\zeta)$, tangent to $M^{\prime}$. Thus $f^{p}(\zeta) \in M$. In particular, $f^{p}(1)$ is the line through $p^{\prime}$, tangent to $M^{\prime}$, i.e., $f^{p}(1)=p$.

(e) is obvious from the corresponding property of $f^{p^{\prime}}$ and Remark 7.3. In view of (e), it suffices to prove (d) for $\zeta=1$, which is easy:

$$
E_{1}^{p}=T_{p}^{1,0} p^{\prime}=H_{p}^{1,0} M \subset \mathbb{C} \otimes T_{p} M .
$$

In (f), smoothness of $F(p, \zeta)$ has already been mentioned. Next, observe that since $E_{0}^{p^{\prime}} \subset T_{0}^{1,0} \mathbb{P}_{2}^{*}, \rho\left(E_{0}^{p^{\prime}}\right) \in \mathbb{P}_{2}$ is a point that corresponds under (8.1) to a line $\ell \subset \mathbb{P}_{2}^{*}$ through $0^{\prime}$; hence $\rho\left(E_{0}^{p^{\prime}}\right)$ is on the line at infinity $0^{\prime}=\mathbb{P}_{2} \backslash \mathbb{C}^{2}$ :

$$
F(p, 0)=f^{p}(0)=\rho\left(E_{0}^{p^{\prime}}\right) \in 0^{\prime} .
$$

Also

$$
E_{0}^{p}=\varepsilon\left(f^{p}(0), f^{p^{\prime}}(0)\right)=\varepsilon\left(f^{p}(0), 0^{\prime}\right)=T_{f^{p}(0)}^{1,0} 0^{\prime} .
$$

Therefore $F_{*}\left(T_{p} M \times\{0\}\right) \subset T_{f^{p}(0)} 0^{\prime} \subset E_{0}^{p} \oplus \bar{E}_{0}^{p}$ holds. 
It remains to prove (c) and the (related) facts that $f^{p}$ and $F(\cdot, \zeta)(\zeta \neq 0)$ are immersions. We are going to show that the restriction of $F$ to $M \times(0,1]$ is a diffeomorphism; combined with (e) this will then take care of those facts.

We have already established that the mapping

$$
F^{\prime}\left(p^{\prime}, \zeta\right)=f^{p^{\prime}}(\zeta) \quad\left(p^{\prime} \in M, \zeta \in(0,1]\right)
$$

is a diffeomorphism, see formula (7.9) and after. To connect $F$ with $F^{\prime}$ it will be convenient to introduce the Green function of $D$ associated with the Monge-Ampère operator.

Define $u^{\prime}: \bar{D}^{\prime} \rightarrow[-\infty, 0]$ by $u^{\prime}\left(q^{\prime}\right)=\log |\zeta|^{2}$ if $q^{\prime}=f^{p^{\prime}}(\zeta)$ for some $p^{\prime} \in M^{\prime}$. In [21] it was proved that $u^{\prime}$ is smooth on $\bar{D}^{\prime} \backslash\left\{0^{\prime}\right\}$, and satisfies

$$
\begin{cases}u^{\prime} \text { is plurisubharmonic } & \text { on } D^{\prime}, \\ \left(\partial \bar{\partial} u^{\prime}\right)^{2}=0 & \text { on } D^{\prime} \backslash\left\{0^{\prime}\right\}, \\ u^{\prime}=0 & \text { on } M^{\prime}, \\ u^{\prime}\left(q^{\prime}\right)=2 \log \operatorname{dist}\left(0^{\prime}, q^{\prime}\right)+O(1) & \text { as } q^{\prime} \rightarrow 0^{\prime}\end{cases}
$$

(Théorème 4). Furthermore, with $p^{\prime} \in M^{\prime}$ and corresponding $f^{p^{\prime}}, E^{p^{\prime}}$, the form $\sigma^{\prime}$ defined by (7.1) (with primes added; cf. also (7.7) and (7.8)) satisfies

$$
\sigma^{\prime}(\zeta) / \zeta=\partial u^{\prime}\left(f^{p^{\prime}}(\zeta)\right) \quad(\zeta \in \bar{\Delta} \backslash\{0\}) .
$$

(This is observed at the end of Chapter 8 in [21]. Thus

$$
E_{\zeta}^{p^{\prime}}=\operatorname{Ker} \partial u^{\prime}\left(f^{\prime}(\zeta)\right) \quad(\zeta \in \bar{\Delta} \backslash\{0\}) .
$$

In Chapters 3 and 5 in [24] a mapping $\gamma_{u^{\prime}}: \bar{D}^{\prime} \backslash\left\{0^{\prime}\right\} \rightarrow \mathbb{P}_{2}$ was associated with the function $u^{\prime}$ as above. If $q^{\prime} \in \bar{D}^{\prime} \backslash\left\{0^{\prime}\right\}$ then $\gamma_{u^{\prime}}\left(q^{\prime}\right)$ is the line in $\mathbb{P}_{2}^{*}$ that is tangent to the level hypersurface $\left\{u^{\prime}=u^{\prime}\left(q^{\prime}\right)\right\}$. Under identification (8.1), $\gamma_{u^{\prime}}\left(q^{\prime}\right)$ can be regarded as a point in $\mathbb{P}_{2}$. Clearly

$$
\gamma_{u^{\prime}}\left(q^{\prime}\right)=\rho\left(\operatorname{Ker} \partial u^{\prime}\left(q^{\prime}\right)\right)
$$

so that by (8.2) and (8.3)

$$
f^{p}(\zeta)=\gamma_{u^{\prime}}\left(f^{p^{\prime}}(\zeta)\right), \quad \zeta \in \bar{\Delta} \backslash\{0\},
$$

or

$$
F(p, \zeta)=\gamma_{u^{\prime}}\left(F^{\prime}\left(p^{\prime}, \zeta\right)\right), \quad \zeta \in(0,1] .
$$

Since by [24, p. 882] $\gamma_{u^{\prime}}$ is a diffeomorphism between $\bar{D}^{\prime} \backslash\left\{0^{\prime}\right\}$ and $\mathbb{C}^{2} \backslash D$, (8.6) implies that $F$ restricted to $M \times(0,1]$ is a diffeomorphism onto $\mathbb{C}^{2} \backslash D$ as claimed. (We remark here that in $[23,24]$ we treated only analytically bounded domains $D$. However, the proofs, with obvious modifications, carry over to smooth domains.)

Thus all assumptions of Lemma 7.1 are satisfied. It remains to decide if the $S^{1}$ action $\left\{g_{\zeta}\right\}$ defined there is positive or not. This can be done exactly as in the first part of this proof, in $\S 7$; the only difference being that 
since $D$ now is replaced by its complement, the defining function $-v$ becomes plurisuperharmonic. As a consequence, $\left\{g_{\zeta}\right\}$ does not turn out positive; therefore the reverse action $\left\{g_{\zeta^{-1}}\right\}$ is an outer action.

Remark 8.3. The (free) inner actions on linearly convex hypersurfaces $M$ constructed in $\S 7$ do not depend on the particular embedding of $M$ in $\mathbb{C}^{2}$. The (free) outer actions constructed in this section, on the contrary, do, because, in general, to different embeddings of $M$ holomorphically inequivalent duals $M^{\prime}$ correspond. Since the space of (linearly convex) embeddings of $M$ is, loosely speaking, parametrized by pairs of functions of two variables, it follows that the parameter space of free outer actions on $M$ is infinite dimensional. This should be compared with Theorem 6.4.

There is one more idea, related to the previous construction, that we want to explain here. The indicatrix $\bar{I}=\bar{I}_{D}$ of a strictly linearly convex domain $D$ with respect to a point $0 \in D$ is defined by

$$
\bar{I}=\left\{f_{*}(0) \frac{\partial}{\partial \zeta} \mid f: \Delta \rightarrow D \text { is holomorphic, } f(0)=0\right\} .
$$

$\bar{I}$ is a closed, strictly convex, circular domain in $T_{0}^{1,0} D$; we shall mostly think of it as lying in $\mathbb{C}^{2} \cong T_{0}^{1,0} D$. The interior of $\bar{I}$ will be denoted $I$. The circular representation is a smooth mapping $\Psi: \bar{I} \backslash\{0\} \rightarrow \bar{D} \backslash\{0\}$, defined by $\Psi(v)=f(\lambda)$ if $f$ is an extremal mapping such that $\lambda f_{*}(0) \frac{\partial}{\partial \zeta}=v(0<\lambda \leq 1)$ (see [31]). $\Psi$ does not extend smoothly to 0 , but if $\hat{\bar{I}}$ and $\hat{\bar{D}}$ denote the blowups of $\bar{I}$, resp. $\bar{D}$, at $0, \Psi$ does extend to a diffeomorphism between $\hat{\bar{I}}$ and $\hat{\bar{D}}$ (this follows from Lemmas 5 and 6 in [23]).

An "exterior" version of this is the following. Given $\bar{D}$ as above, construct its dual $\bar{D}^{\prime}$, the indicatrix $\bar{I}_{D^{\prime}}\left(\right.$ at $\left.0^{\prime}\right)$, and the dual $\bar{I}_{D^{\prime}}^{\prime}$ of $\bar{I}_{D^{\prime}}$. With $u_{0}$ denoting the solution of the Monge-Ampère equation (8.3) with $D$ replaced by $I_{D^{\prime}}$ (and $M$ by $\partial I_{D^{\prime}}$ ), we get three diffeomorphisms

$$
\mathbb{C}^{2} \backslash D \stackrel{\gamma_{u^{\prime}}}{\longleftarrow} \bar{D}^{\prime} \backslash\left\{0^{\prime}\right\} \stackrel{\Psi}{\longleftarrow} \bar{I}_{D^{\prime}} \backslash\left\{0^{\prime}\right\} \stackrel{\gamma_{u_{0}}}{\longrightarrow} \mathbb{C}^{2} \backslash I_{D^{\prime}}^{\prime}
$$

$u_{0}$ and $\gamma_{u_{0}}$ can be computed more or less explicitly and one finds that $\gamma_{u_{0}}$ extends to a diffeomorphism $\hat{\bar{I}}_{D^{\prime}} \rightarrow \mathbb{P}_{2} \backslash I_{D^{\prime}}^{\prime}$. More generally, using the precise description of extremal discs given in Lemmas 5,6 in [23], and (8.4), one can check that also $\gamma_{u^{\prime}}$ extends to a diffeomorphism $\hat{\bar{D}}^{\prime} \rightarrow \mathbb{P}_{2} \backslash D$. Thus we obtain a diffeomorphism

$$
\Theta: \mathbb{P}_{2} \backslash I_{D^{\prime}}^{\prime} \rightarrow \mathbb{P}_{2} \backslash D
$$

an exterior analog of the circular representation. Its main feature is that if $L$ is a linear mapping $\mathbb{C} \rightarrow \mathbb{C}^{2}$, then for some $r \Theta(L(r / \zeta))$ is an "extremal" mapping $f^{p}$ constructed in the previous proof. Also $\Theta$ is equivariant between the standard $S^{1}$ action

$$
\partial I_{D^{\prime}}^{\prime} \ni\left(z_{1}, z_{2}\right) \mapsto\left(\zeta z_{1}, \zeta z_{2}\right) \in \partial I_{D^{\prime}}^{\prime} \quad\left(\zeta \in S^{1}\right)
$$

and the outer $S^{1}$ action on $M$ constructed above. 
9. Attaching a pseudoconcave surface to a CR manifold. The aim of this and the next section is to prove Theorem 4.6, and also to prepare the proof of the stability theorem (Theorem 4.5). Let $M_{0} \subset \mathbb{C}^{2}$ be a connected, compact, strictly pseudoconvex hypersurface. $M_{0}$ cuts the projective plane $\mathbb{P}_{2} \supset \mathbb{C}^{2}$ in two; denote by $D_{0}$ the component of $\mathbb{P}_{2} \backslash M_{0}$ that contains the line at infinity, $\mathbb{P}_{1}(\infty)$. Then $\bar{D}_{0}$ is a compact strictly pseudoconcave surface with boundary $M_{0}$. Now let $M$ be a small perturbation of the CR manifold $M_{0}$ as discussed in $\S 4$. It turns out that then the complex structure of $\bar{D}_{0}$ can be perturbed so that the resulting complex surface $\bar{D}$ has boundary $M$.

Fixing a Riemannian metric on $M_{0}$ and $\bar{D}_{0}$, for any positive integer $k$ we can talk about the $C^{k}$ distance of a CR perturbation $M$ to $M_{0}$ and also about the $C^{k}$ distance of a perturbed complex structure $\bar{D}$ to $\bar{D}_{0}$. Then we have the following theorem:

Theorem 9.1. Let $M_{0}, \bar{D}_{0}$ be as above, and let $a \geq 3$ be an integer, $\eta a$ positive number. Then there exists a positive $\varepsilon$ with the following property. For any perturbation $M$ of the $C R$ structure of $M_{0}$ whose $C^{36 a+213}$ distance to $M_{0}$ is less than $\varepsilon$ there is a perturbation $\bar{D}$ of the complex structure of $\bar{D}_{0}$ such that

(i) the boundary of $\bar{D}$ is $\bar{M}$;

(ii) the almost complex tensors of $\bar{D}$ and $\bar{D}_{0}$ agree in points of $\mathbb{P}_{1}(\infty)$, the line at infinity in $D_{0}$. (In particular $\mathbb{P}_{1}(\infty)$ is a complex submanifold in $D)$;

(iii) the $C^{a}$ distance between the complex structures of $\bar{D}$ and $\bar{D}_{0}$ is less than $\eta$.

Our original proof of this theorem (with different constants) worked only for strictly linearly convex $M_{0}$. The following approach has been suggested to us by Catlin.

The Fubini-Study metric on $\bar{D}_{0} \subset \mathbb{P}_{2}$ endows the holomorphic vector bundle $T^{1,0} D_{0}$ with a hermitian metric, and the space of its $L^{2}$ sections with the structure of a Hilbert space. More generally, for $q=0,1,2$ the space of $(0, q)$ forms on $D_{0}$ whose coefficients are $L^{2}$ sections of $T^{1,0} D_{0}$ constitute a Hilbert space $\mathscr{H}_{q}$. Furthermore, denote by $E$ the line bundle over $\mathbb{P}_{2}$ defined by the divisor $\mathbb{P}_{1}(\infty)$. This again has a hermitian structure; denote by $\mathscr{K}_{q}$ the Hilbert space of $(0, q)$ forms on $D_{0}$ whose coefficients are $L^{2}$ sections of $T^{1,0}\left(D_{0}\right) \otimes E^{-1}$.

Restrict $\bar{\partial}$ to smooth forms in $\mathscr{H}_{q}$ (resp. $\mathscr{K}_{q}$ ) that are compactly supported in $D_{0}$, and denote the adjoint of this operator by $\bar{\partial}_{0}^{*}: \mathscr{H}_{q+1} \rightarrow \mathscr{H}_{q}$ (resp. $\left.\bar{\partial}_{0}^{*}: \mathscr{K}_{q+1} \rightarrow \mathscr{K}_{q}\right)$. Next, let $\bar{\partial}_{0}$ denote the adjoint of $\bar{\partial}_{0}^{*}$. Then $\bar{\partial}_{0}^{*}$ is the adjoint of $\bar{\partial}_{0}$.

Proposition 9.2. If $u \in \mathscr{H}_{2}$ (resp. $u \in \mathscr{K}_{2}$ ) satisfies $\bar{\partial}_{0}^{*} u=0$ then $u=0$.

In other words, the compactly supported cohomology groups $H_{c}^{2}\left(D_{0}, T^{1,0} D_{0}\right)$ and $H_{c}^{2}\left(D_{0}, T^{1,0} D_{0} \otimes E^{-1}\right)$ vanish, cf. [14]. 
Proof. If $F \rightarrow X$ is a holomorphic vector bundle with hermitian metric ( , ) over a hermitian manifold $X$, and $F^{*}$ is its dual bundle, then there is a conjugate linear mapping $\psi \mapsto \psi^{*}$ from $F$-valued $(p, q)$ forms to $F^{*}$-valued $(n-p, n-q)$ forms, given by

$$
\phi \wedge \psi^{*}=(\phi, \psi) \text { times volume form of } X
$$

for $\phi, \psi \quad F$ valued $(p, q)$ forms. If $\bar{\partial}^{*}$ denotes the formal adjoint of $\bar{\partial}$ acting on $F$ valued $(p, q)$ forms then

$$
\bar{\partial} \psi^{*}=(-1)^{p+q}\left(\bar{\partial}^{*} \psi\right)^{*}
$$

(see, e.g., [16]). Specializing to our case of, say, $\psi=u \in \mathscr{R}_{2}$ with $\bar{\partial}_{0}^{*} u=0$ we get $\bar{\partial} u^{*}=0$; i.e., $u^{*}$ is a holomorphic $(2,0)$ form with values in the bundle $T^{1,0}\left(D_{0}\right)^{*} \otimes E$.

The vector field given by $\partial / \partial z_{j}, \quad(j=1,2)$ on the affine part $\mathbb{C}^{2} \subset \mathbb{P}_{2}$ extends to $\mathbb{P}_{1}(\infty)$ and vanishes there, so that it can be regarded as a holomorphic section of $T^{1,0}\left(D_{0}\right) \otimes E^{-1}$. Pairing it with $u^{*}$ we get a scalar valued holomorphic $(2,0)$ form $v_{j}$ on $D_{0}$, which by Hartogs' theorem holomorphically extends to $\mathbb{P}_{2}$. It follows that $v_{j}=0$ and therefore $u=0$.

The case of $u \in \mathscr{H}_{2}$ is treated in the same way.

Proof of Theorem 9.1. Recall Kiremidjian's theorem specialized to the case of our two-dimensional complex surface $\bar{D}_{0}$ with strictly pseudoconcave boundary $M_{0}$ (see [14]). Under the vanishing property expressed by Proposition 9.2 (for $\mathscr{H}_{2}$ ), somewhat loosely stated it says that small CR perturbations $M$ of $M_{0}$ all arise as boundaries of small perturbations $\bar{D}$ of the complex manifold $\bar{D}_{0}$. More precisely, tracking down the constants $k, \lambda, \lambda_{0}, \mu$ in the proof of Theorem 3.3 in [14], we get our Theorem 9.1, except for property (ii) of $\bar{D}$. To arrange that the complex structure of $\bar{D}$ in points of $\mathbb{P}_{1}(\infty)$ does not change, a mild modification of the construction in [14] will be needed. Namely, instead of $T^{1,0} \bar{D}_{0}$-valued forms (in the notation of [14] $T^{\prime}(\bar{M})$-valued forms) one should work with $T^{1,0} \bar{D}_{0} \otimes E^{-1}$-valued forms. These forms can also be thought of as certain $T^{1,0} \bar{D}_{0}$-valued forms that vanish on $\mathbb{P}_{1}(\infty)$ : if $\sigma$ is a holomorphic section of $E$ whose divisor is $\mathbb{P}_{1}(\infty)$ then multiplication by $\sigma$ embeds the space of $T^{1,0} \bar{D}_{0} \otimes E^{-1}$-valued forms into a subspace of $T^{1,0} \bar{D}_{0}$-valued forms vanishing at $\mathbb{P}_{1}(\infty)$.

Now in view of the vanishing property expressed by Proposition 9.2 for the space $\mathscr{K}_{2}$, in Kiremidjian's proof $T^{1,0} \bar{D}_{0}$ can be replaced by $T^{1,0} \bar{D}_{0} \otimes E^{-1}$, and one gets that his equation (3.15) in [14] can be solved with $\omega$ that vanishes at $\mathbb{P}_{1}(\infty)$. This $\omega$ then defines a complex manifold structure $\bar{D}$ on $\bar{D}_{0}$ which agrees with that of $\bar{D}_{0}$ in points of $\mathbb{P}_{1}(\infty)$, and whose boundary is $M$.

10. The existence of outer actions. Now let $M_{0} \subset \mathbb{P}_{2}$ denote the boundary of a strictly linearly convex domain and let $D_{0}$ denote the pseudoconcave component of $\mathbb{P}_{2} \backslash M_{0}$. Let $M$ be a small perturbation of $M_{0}$ as in Theorem 4.6. Then by Theorem 9.1 it bounds a small perturbation $\bar{D}$ of $\bar{D}_{0}$; moreover, the 
almost complex tensors of $\bar{D}$ and $\bar{D}_{0}$ can be assumed to agree in points of $\mathbb{P}_{1}(\infty)$. We shall deduce the existence of an outer action on $M$ by constructing mappings $f=f^{p}$ and line bundles $E=E^{p}(p \in M)$ as in Lemma 7.1.

Theorem 10.1. Let $\bar{D}_{0}$ and $\bar{D}$, a sufficiently small perturbation of $\bar{D}_{0}$, be as above. Then for every $p \in M$ there are a smooth embedding $f=f^{p}: \bar{\Delta} \rightarrow \bar{D}$ and a smooth rank one subbundle $E=E^{p}$ of $f^{*} T^{1,0} \bar{D}$ such that

(a) $f$ and $E$ are holomorphic on $\Delta$;

(b) $f(1)=p, f\left(S^{1}\right) \subset M, f(0) \in \mathbb{P}_{1}(\infty)$;

(c) for every $\zeta \in \bar{\Delta}, f_{*}\left(T_{\zeta}^{1,0} \bar{\Delta}\right)$ and $E_{\zeta}$ are transverse;

(d) for $\zeta \in S^{1}, E_{\zeta}$ is tangent to $M, E_{0}$ is tangent to $\mathbb{P}_{1}(\infty)$;

(e) if $P=f^{p}(\omega)$ for some $\omega \in S^{1}$ then $f^{P}(\zeta)=f^{p}(\zeta \omega)$;

(f) putting $F(p, \zeta)=f^{p}(\zeta), F: M \times \bar{\Delta} \rightarrow \bar{D}$ is smooth and, for fixed $\zeta \in \bar{\Delta} \backslash\{0\}, F(\cdot, \zeta): M \rightarrow \bar{D}$ is an immersion.

Compared with the assumptions of Lemma 7.1 the following difference is observed. Here we are not explicitly claiming $F_{*}\left(T_{p} M \times\{0\}\right) \subset E_{0}^{p} \oplus \bar{E}_{0}^{p}$. Nevertheless, this follows from (b) (whence $\left.F(M \times\{0\}) \subset \mathbb{P}_{1}(\infty)\right)$ and (d). Therefore Theorem 4.6 will follow if we can prove Theorem 10.1 .

Observe that when $D=D_{0}$, the existence of such $f$ and $E$ has been proved (see $\S 8)$. In fact, in this case $F(\cdot, \zeta)$ is an embedding for $\zeta \in \bar{\Delta} \backslash\{0\}$. Call $f, E$ corresponding to $D=D_{0} f$ and ${ }_{0} E$. It will be advantageous to put these two kinds of objects on the same footing. In order to do so, consider the projectivization $\mathbb{P}^{*} T^{1,0} \bar{D}_{0} \rightarrow \bar{D}_{0}$ of the vector bundle $T^{1,0} \bar{D}_{0}$, consisting of lines in $T^{1,0} \bar{D}_{0}$. Thus $\mathbb{P}^{*} T^{1,0} \bar{D}_{0}=\bar{Q}$ is a three-dimensional compact complex manifold with boundary and it is a smooth fiber bundle over $\bar{D}_{0}$, the projection

$$
\Pi: \bar{Q} \rightarrow \bar{D}_{0}
$$

being holomorphic over the interior $Q$ of $\bar{Q} \cdot M_{0}$ lifts to a compact smooth submanifold $\widetilde{M}_{0} \subset \partial \bar{Q}$

$$
\widetilde{M}_{0}=\left\{H_{p}^{1,0} M_{0}: p \in M_{0}\right\} \subset \mathbb{P}^{*}\left(T^{1,0} \bar{D}_{0}\right) .
$$

$\mathbb{P}_{1}(\infty) \subset \bar{D}_{0}$ also lifts, to a complex curve

$$
C_{0}=\left\{T_{p}^{1,0} \mathbb{P}_{1}(\infty): p \in \mathbb{P}_{1}(\infty)\right\} \subset Q .
$$

Given now a ${ }_{0} f={ }_{0} f^{p},{ }_{0} E={ }_{0} E^{p}$ as in Theorem 10.1 (with $\bar{D}=\bar{D}_{0}$ ), we can lift this pair to a mapping $g_{0}=g_{0}^{p}: \bar{\Delta} \rightarrow \bar{Q}$ by putting

$$
g_{0}(\zeta)={ }_{0} E_{\zeta} \in \mathbb{P}^{*}\left(T^{1,0} \bar{D}_{0}\right)
$$

Proposition 10.2. $g_{0}$ is holomorphic in $\Delta, g_{0}\left(S^{1}\right) \subset \widetilde{M}_{0}$, and $g_{0}(0) \in C_{0}$. Furthermore, $g_{0}$ is transverse to $\partial Q$.

Indeed, this follows since ${ }_{0} f$ and ${ }_{0} E$ enjoy the properties (a), (b), (c) and (d) in Theorem 10.1. 
We shall now prove that if the complex structure of $\bar{Q}$ is perturbed a little, there will still be mappings $g$ of $\bar{\Delta}$ into the perturbed complex manifold that have corresponding properties. Analytically it will be convenient to define a new (almost) complex structure on $\bar{Q}$ by a $T^{1,0} \bar{Q}$-valued $(0,1)$ form $\Omega$. If $\Omega$ is such a form, sufficiently small, then by declaring vectors of form

$$
\bar{X}-\Omega(\bar{X}), \quad \bar{X} \in T^{0,1} \bar{Q},
$$

to be $(0,1)$ vectors, we get a new almost complex structure on $\bar{Q}$. This new almost complex manifold will be denoted $\bar{Q}_{\Omega}$. Conversely, any small perturbation of the complex structure of $\bar{Q}$ can be described by an $\Omega$ as above.

Given a smooth mapping $g: \bar{\Delta} \rightarrow \bar{Q}$, decompose its restricted differential $\left.g_{*}\right|_{T^{0,1} \bar{\Delta}}$ as $\bar{\partial} g+\partial g$ where $\bar{\partial} g$, resp. $\partial g$, are the $T^{1,0} \bar{Q}$, resp. $T^{0,1} \bar{Q}$, components. Thus $\bar{\partial} g(\zeta): T_{\zeta}^{0,1} \bar{\Delta} \rightarrow T_{g(\zeta)}^{1,0} \bar{Q}$, and $g$ is holomorphic if $\bar{\partial} g \equiv 0$. The same $g$, considered as a mapping into $Q_{\Omega}$, is holomorphic if and only if

$$
\bar{\partial}_{\Omega} g \equiv \bar{\partial} g+\Omega(\partial g)=0 \text {. }
$$

To get solutions of this equation satisfying certain boundary and initial conditions we shall have to introduce certain Banach spaces. First endow $\mathbb{P}^{*}\left(T^{1,0} \mathbb{P}_{2}\right)$ with a smooth Kähler metric, and choose an integer $b \geq 2$. Using the restriction of the metric to $\bar{Q} \subset \mathbb{P}^{*}\left(T^{1,0} \mathbb{P}_{2}\right)$ and the covariant differentiation it determines, one obtains a Banach space structure on the space of $b$ times continuously differentiable $T^{1,0} \bar{Q}$-valued $(0,1)$ forms on $\bar{Q}$. Since such forms determine almost complex structures on $\bar{Q}$, we shall denote this Banach space $\operatorname{Str}_{b}$. Furthermore, consider the set of those mappings $g: \bar{\Delta} \rightarrow \bar{Q}$ that have Hölder continuous first derivatives of exponent, say $\frac{1}{2}$, (i.e., are $\frac{3}{2}$-Hölder continuous); that are transverse to $\partial Q$; and satisfy

$$
\begin{gathered}
g\left(S^{1}\right) \subset \widetilde{M}_{0}, \\
g(0) \in C_{0} .
\end{gathered}
$$

Such mappings constitute a smooth Banach manifold to be denoted Map. The set $\left\{g_{0}^{p}: p \in M\right\}=K$ is a compact subset of Map.

Lemma 10.3. There are a neighborhood $U_{1}$ of $0 \in \operatorname{Str}_{b}$, a neighborhood $U_{2}$ of $K \subset$ Map and a mapping

$$
\Phi: U_{1} \times \widetilde{M}_{0} \rightarrow \text { Map }
$$

of class $C^{b-1}$ such that

(a) for $\tilde{p} \in \widetilde{M}_{0} \quad \Phi(0, \tilde{p})=g_{0}^{p}$, where $p=\Pi \tilde{p} \in M_{0}$;

(b) for $\Omega \in U_{1}, \tilde{p} \in \widetilde{M}_{0} \Phi(\Omega, \tilde{p})=g$ satisfies (10.4); i.e. it is a holomorphic mapping into $Q_{\Omega}$, and $g(1)=\tilde{p}$;

(c) conversely, if $g \in U_{2}$ satisfies (10.4) with $\Omega \in U_{1}$ then $g=\Phi(\Omega, \tilde{p})$ with $\tilde{p}=g(1)$. Furthermore, if $\Omega \in U_{1}$ is infinitely differentiable then the mapping 


$$
\Theta: \widetilde{M}_{0} \ni \tilde{p} \mapsto \Phi(\Omega, \widetilde{p}) \in \operatorname{Map}
$$

itself is infinitely differentiable.

We shall apply the implicit function theorem to prove this lemma, but first we need to introduce more notation. For $q_{1}, q_{2} \in \bar{Q}$ nearby points let $I\left(q_{1}, q_{2}\right)$ : $T_{q_{1}}^{1,0} \bar{Q} \rightarrow T_{q_{2}}^{1,0} Q$ denote parallel transport along the shortest geodesic (in $\left.\mathbb{P}^{*}\left(T^{1,0} \mathbb{P}_{2}\right)\right)$ joining $q_{1}, q_{2} . I\left(q_{1}, q_{2}\right)$ depends smoothly on $q_{1}, q_{2}$. If $g, g_{0} \in$ Map are nearby mappings, let $I_{g_{0}} \bar{\partial}_{\Omega} g$ denote the $g_{0}^{*} T^{1,0} \bar{Q}$-valued $(0,1)$ form on $\bar{\Delta}$ defined by

$$
I_{g_{0}} \bar{\partial}_{\Omega} g(\zeta)=I\left(g(\zeta), g_{0}(\zeta)\right) \bar{\partial}_{\Omega} g(\zeta) \quad(\zeta \in \bar{\Delta}) .
$$

Finally, for $g_{0} \in$ Map, denote by $B=B_{g_{0}}$ the Banach space of $g_{0}^{*} T^{1,0} \bar{Q}$ valued $(0,1)$ forms on $\bar{\Delta}$ which are Hölder continuous of exponent $\frac{1}{2}$.

Proof of Lemma 10.3. I. It suffices to construct $\Phi$ on some neighborhood $U_{1} \times$ $U_{3}$ of an arbitrary point $\left(0, \widetilde{p}_{0}\right) \in \operatorname{Str}_{b} \times \widetilde{M}_{0}$ Putting $p_{0}=\Pi \widetilde{p}_{0}$ and $g_{0}=g_{0}^{p_{0}}$, (c) of this lemma should then be understood with $U_{2}$ a neighborhood of $g_{0}$. Now fix $\widetilde{p}_{0}$ and with it, $g_{0}$; then $g_{0}(1)=\widetilde{p}_{0}$.

With $B=B_{g_{0}}$ as above define a mapping $\Psi$ from a neighborhood of $\left(0, g_{0}\right) \in \operatorname{Str}_{b} \times$ Map to $B \times \widetilde{M}_{0}$ by

$$
\Psi(\Omega, g)=\left(I_{g_{0}} \bar{\partial}_{\Omega} g, g(1)\right) \text {. }
$$

Then $\Psi$ is of class $C^{b-1}$. The existence of $\Phi$ (near $\left.\left(0, \tilde{p}_{0}\right)\right)$ would follow if we could prove that the partial derivative

$$
L_{0}=\frac{\partial \Psi}{\partial g}\left(0, g_{0}\right): T_{g_{0}} \operatorname{Map} \rightarrow B \times T_{\widetilde{p}_{0}} \widetilde{M}
$$

is an isomorphism. Here $T_{g_{0}}$ Map is a subspace of the space of $\frac{3}{2}$-Hölder continuous sections of $g_{0}^{*} T \bar{Q}$. We shall pass from $g_{0}^{*} T \bar{Q}$ to the bundle $g_{0}^{*} T^{1,0} \bar{Q}$, which has the advantage of being holomorphic (over $\Delta$ ). Otherwise the two bundles are $\mathbb{R}$-isomorphic via

$$
g_{0}^{*} T^{1,0} \bar{Q} \ni X \mapsto \operatorname{Re} X \in g_{0}^{*} T \bar{Q}
$$

If $h$ is a $\frac{3}{2}$-Hölder continuous section of $g_{0}^{*} T^{1,0} \bar{Q}$ then (10.5), (10.6) yield that $\operatorname{Re} h \in T_{g_{0}}$ Map if and only if

$$
\begin{gathered}
\operatorname{Re} h(\zeta) \in T_{g_{0}(\zeta)} \widetilde{M}_{0} \quad\left(\zeta \in S^{1}\right), \\
h(0) \in T_{g_{0}(0)}^{1,0} C_{0}
\end{gathered}
$$

hold. Let $A$ denote the Banach space of such sections $h$ (satisfying (10.9), (10.10)). It will suffice to show that the mapping

is an isomorphism.

$$
L: A \ni h \mapsto L_{0}(\operatorname{Re} h) \in B \times T_{\widetilde{p}_{0}} \widetilde{M}_{0}
$$


It is readily computed (in local coordinates, for example) that for $h \in A$

$$
L h=(\bar{\partial} h, \operatorname{Re} h(1)) .
$$

To check the invertibility of $L$ (with boundary conditions (10.9), (10.10)) is not straightforward, and uses strict linear convexity in an essential way. To make $L$ analytically accessible, we shall introduce local coordinates, and trivialize the bundle $g_{0}^{*} T^{1,0} \bar{Q}$.

II. First introduce homogeneous coordinates $\left(Z_{0}: Z_{1}: Z_{2}\right)$ on $\mathbb{P}_{2} \supset \bar{D}_{0}$ so that $\mathbb{P}_{1}(\infty)=\left\{Z_{0}=0\right\}$. We can assume that ${ }_{0} f(0)=\Pi g_{0}(0)=(0: 1: 0)$. Since the embeddings $f={ }_{0} f$ constructed in $\S 8$ had the property that $f(\zeta) \notin$ $\mathbb{P}_{1}(\infty)$ if $\zeta \neq 0$, we can write

$$
{ }_{0} f(\zeta)=\left(\zeta: f_{1}(\zeta): f_{2}(\zeta)\right)
$$

with $f_{1}, f_{2}$ holomorphic on $\Delta$ and smooth on $\bar{\Delta}$ with $f_{2}(0)=0 \neq f_{1}(0)$.

We shall also need a coordinate $w$ on the fibers of $\Pi: \mathbb{P}^{*}\left(T^{1,0} \mathbb{P}_{2}\right) \rightarrow \mathbb{P}_{2}$ (near $\left.g_{0}(\bar{\Delta})\right)$. Let $X$ denote any smooth, nonvanishing section of ${ }_{0} E$, holomorphic over $\Delta$. Since the vector fields $Z_{0} \frac{\partial}{\partial Z_{1}}$ and $Z_{0} \frac{\partial}{\partial Z_{2}}$ are well defined on $\mathbb{P}_{2}$ and in points $q \in \mathbb{P}_{2} \backslash \mathbb{P}_{1}(\infty)$ span $T_{q}^{1,0} \mathbb{P}_{2}$, we can write

$$
X(\zeta)=a_{1}(\zeta) Z_{0} \frac{\partial}{\partial Z_{1}}+a_{2}(\zeta) Z_{0} \frac{\partial}{\partial Z_{2}}
$$

with $a_{1}, a_{2}$ smooth on $\bar{\Delta} \backslash\{0\}$, holomorphic on $\Delta \backslash\{0\}$.

Proposition 10.4. $a_{1}$ and $a_{2}$ have at most a simple pole at 0 , with $\operatorname{Res}_{0} a_{2} \neq 0$. Proof. In a neighborhood of $(0: 1: 0)$ the fields $Z_{1} \frac{\partial}{\partial Z_{0}}$ and $Z_{1} \frac{\partial}{\partial Z_{2}}$ are independent. In view of $\sum_{j=0}^{2} Z_{j} \frac{\partial}{\partial Z_{j}}=0$ we obtain for $\zeta$ near 0

$$
\begin{aligned}
X(\zeta) & =-a_{1}(\zeta) \frac{Z_{0}^{2}}{Z_{1}} \frac{\partial}{\partial Z_{0}}+\left(a_{2}(\zeta) Z_{0}-a_{1}(\zeta) \frac{Z_{0} Z_{2}}{Z_{1}}\right) \frac{\partial}{\partial Z_{2}} \\
& =a_{1}(\zeta) \frac{\zeta^{2}}{f_{1}^{2}(\zeta)} Z_{1} \frac{\partial}{\partial Z_{0}}+\left(a_{2}(\zeta) \frac{\zeta}{f_{1}(\zeta)}-a_{1}(\zeta) \frac{\zeta f_{2}(\zeta)}{f_{1}^{2}(\zeta)}\right) Z_{1} \frac{\partial}{\partial Z_{2}}
\end{aligned}
$$

where we have also used (10.12). Since $X(0)$ is tangential to $\mathbb{P}_{1}(\infty)=$ $\left\{Z_{0}=0\right\}$, we must have

$$
\lim _{\zeta \rightarrow 0} \zeta^{2} a_{1}(\zeta)=0
$$

whence $a_{1}$ has a simple pole at 0 . This implies

$$
\lim _{\zeta \rightarrow 0} a_{1}(\zeta) \frac{\zeta f_{2}(\zeta)}{f_{1}^{2}(\zeta)}=0
$$

Finally, because $X(0) \neq 0$ we have that $\lim _{\zeta \rightarrow 0} \zeta a_{2}(\zeta)$ exists and is nonzero.

It follows that for small $\varepsilon>0$ a mapping of $\bar{\Delta} \times\{\omega \in \mathbb{C}:|\omega| \leq \varepsilon\}$ into $\mathbb{P}_{2}$ can be defined by

$$
\mu(\zeta, \omega)=\left(\zeta: f_{1}(\zeta)+\omega \zeta a_{1}(\zeta): f_{2}(\zeta)+\omega \zeta a_{2}(\zeta)\right)
$$


Since $\mu_{*}(\zeta, 0)\left(\frac{\partial}{\partial \omega}\right)=X(\zeta)$, which is transverse to ${ }_{0} f_{*}(\zeta)\left(\frac{\partial}{\partial \zeta}\right)$, it follows that $\mu$ is a diffeomorphism (if $\varepsilon$ is sufficiently small). Also, restricted to $\Delta \times$ $\{|\omega|<\varepsilon\}, \mu$ is biholomorphic.

Now define smooth $(1,0)$ vector fields $V_{1}, V_{2}$ on a neighborhood of ${ }_{0} f(\bar{\Delta})$ such that on the image of $\mu$

$$
V_{1}=\mu_{*} \frac{\partial}{\partial \zeta}, \quad V_{2}=\mu_{*} \frac{\partial}{\partial \omega}
$$

$V_{1}, V_{2}$ are holomorphic on a neighborhood of ${ }_{0} f(\Delta)$ and on a neighborhood $\mathscr{N}$ of ${ }_{0} f(\bar{\Delta})$ they are transverse.

With the help of $V_{1}, V_{2}$ a coordinate can be defined on the fibers of $\Pi$ : $\mathbb{P}^{*}\left(T^{1,0} \mathbb{P}_{2}\right) \rightarrow \mathbb{P}_{2}$, at least near $g_{0}(\bar{\Delta})$. Namely if $\ell \in \mathbb{P}^{*}\left(T_{q}^{1,0} \mathbb{P}_{2}\right)$ is a line in $T_{q}^{1,0} \mathbb{P}_{2}, q \in \mathscr{N}$, which is spanned by a vector $V \in T_{q}^{1,0} \mathbb{P}_{2}$ then $V=$ $c_{1} V_{1}+c_{2} V_{2}$, and $w=c_{1} / c_{2} \in \mathbb{C} \cup\{\infty\}$ will be a coordinate on the fiber $\Pi^{-1}(q)$. For example, ${ }_{0} E_{\zeta} \in \mathbb{P}^{*}\left(T^{1,0} \mathbb{P}_{2}\right)$ has $w$ coordinate 0 for every $\zeta \in \bar{\Delta}$. Of course, $w$ is not everywhere holomorphic, but, what will be essential to us, $d w=\partial w$ is holomorphic along $g_{0}$ (in fact, in a neighborhood of $g_{0}(\Delta)$ ).

Now it is easy to trivialize $g_{0}^{*} T^{1,0} \bar{Q}$. Define three holomorphic sections $v_{1}, v_{2}, v_{3}$ of it as follows. Let $v_{1}=g_{0^{*}} \frac{\partial}{\partial \zeta}$. Then $\Pi_{*} v_{1}(\zeta)=V_{1}\left({ }_{0} f(\zeta)\right)$. Let $v_{2}(\zeta)$ be any lifting of $V_{2}\left({ }_{0} f(\zeta)\right)$ along $\Pi$ which is smooth on $\bar{\Delta}$, holomorphic on $\Delta$ and satisfies $v_{2}(0) \in T^{1,0} C$. Finally, let $v_{3}$ be the section of the line bundle $\left(g_{0}^{*} \operatorname{Ker} \Pi_{*}\right) \cap\left(g_{0}^{*} T^{1,0} \bar{Q}\right)$ determined by

$$
\left\langle d w, v_{3}\right\rangle \equiv 1 \text {. }
$$

$v_{1}, v_{2}, v_{3}$ are pointwise independent, so that any section $h$ of $g_{0}^{*} T^{1,0} \bar{Q}$ can be written as

$$
h=\sum_{j=1}^{3} h_{j} v_{j} .
$$

III. We shall now express the boundary and initial conditions (10.9) and (10.10) in terms of $h_{j}$. Because of $v_{2}(0) \in T^{1,0} C,(10.10)$ is equivalent to

$$
h_{1}(0)=h_{3}(0)=0 \text {. }
$$

As for (10.9), let $r$ denote a smooth defining function of the linearly convex domain $\mathbb{P}_{2} \backslash \bar{D}_{0}$. In a neighborhood of ${ }_{0} f\left(S^{1}\right)$ the $(1,0)$ vector $\left(V_{2} r\right) V_{1}-$ $\left(V_{1} r\right) V_{2} \neq 0$ is tangent to the level hypersurfaces of $r$. Therefore the $w$ coordinate of $H_{q}^{1,0} M_{0} \in \mathbb{P}^{*}\left(T_{q}^{1,0} \mathbb{P}_{2}\right)\left(q \in M_{0}\right)$ is $-\left(V_{2} r\right) /\left(V_{1} r\right)$. It follows that in coordinates $\left(Z_{0}: Z_{1}: Z_{2}, w\right)$ the equation of $\widetilde{M}_{0}$ is

$$
\left\{\begin{aligned}
r & =0 \\
V_{2} r+w V_{1} r & =0
\end{aligned}\right.
$$

In these formulae $r, V_{1} r, V_{2} r$ are lifted to $\mathbb{P}^{*}\left(T^{1,0} \mathbb{P}_{2}\right)$ by $r\left(Z_{0}: Z_{1}: Z_{2}, w\right)=$ $r\left(Z_{0}: Z_{1}: Z_{2}\right)$, etc. 
Hence (10.9) is equivalent to

$$
\begin{gathered}
\langle d r, \operatorname{Re} h\rangle=0 \quad \text { on } S^{1}, \\
\left\langle d\left(V_{2} r+w V_{1} r\right), \operatorname{Re} h\right\rangle=0 \text { on } S^{1} .
\end{gathered}
$$

Summarizing: invertibility of $L$ means that for any given $g^{*} T^{1,0} \bar{Q}$-valued $(0,1)$ form

$$
u=\sum_{1}^{3} u_{j} \otimes v_{j},
$$

with $u_{j} \frac{1}{2}$-Hölder continuous $(0,1)$ forms on $\bar{\Delta}$, and any vector

$$
Y=\sum_{1}^{3} a_{j} v_{j}(1), \quad a_{j} \in \mathbb{C},
$$

satisfying

$$
\langle d r, \operatorname{Re} Y\rangle=\left\langle d\left(V_{2} r+w V_{1} r\right), \operatorname{Re} Y\right\rangle=0,
$$

there is a unique $h$ as in (10.15), $\frac{3}{2}$-Hölder continuous, satisfying (10.16), (10.17), (10.18), and

$$
\begin{aligned}
& \bar{\partial} h_{j}=u_{j}, \\
& h_{j}(1)=a_{j} .
\end{aligned}
$$

IV. Thus, let $u$ and $Y$ be given as above, and we shall show that the above equations have a unique solution $h_{j} \cdot(10.17)$ is equivalent to

$$
\operatorname{Re} h_{1}(\zeta)\left(V_{1}\left({ }_{0} f(\zeta)\right) r\right)=0, \quad \zeta \in S^{1}
$$

We can assume that the defining function $r$ is such that $r\left({ }_{0} f(\zeta)\right)=|\zeta|^{2}-1$ and also $\left(V_{2} r\right)\left({ }_{0} f(\zeta)\right)=0$ for $\zeta$ near $S^{1}$, in which case

$$
V_{1} r=\left({ }_{0} f_{*} \frac{\partial}{\partial \zeta}\right) r=\frac{\partial r\left({ }_{0} f(\zeta)\right)}{\partial \zeta}=\bar{\zeta}
$$

Hence (10.22) can be written

$$
\operatorname{Re} h_{1}(\zeta) / \zeta=0, \quad \zeta \in S^{1}
$$

If $\varphi_{j}$ denotes a $\frac{3}{2}$-Hölder continuous solution of

$$
\bar{\partial} \varphi_{j}=u_{j}, \quad \varphi_{j}(0)=0 \quad(j=1,2,3)
$$

on $\bar{\Delta}$, then the functions $H_{j}=h_{j}-\varphi_{j}$ are holomorphic on $\Delta$, and (10.24) and the first equation of (10.16) become

$$
\operatorname{Re} H_{1}(\zeta) / \zeta=\operatorname{Re} \varphi_{1}(\zeta) / \zeta, \quad \zeta \in S^{1}
$$

$$
H_{1}(0)=0 \text {. }
$$


Further, (10.21) for $j=1$ means

$$
H_{1}(1)=a_{1}+\varphi_{1}(1)
$$

Since the first equation in $(10.19)$ implies $0=\left\langle d r, \quad \operatorname{Re} a_{1} v_{1}(1)\right\rangle=\operatorname{Re} a_{1}$, it follows that (10.25),(10.26) and (10.27) have a unique holomorphic solution $H_{1}$ which is $\frac{3}{2}$-Hölder continuous. Thus $h_{1}$ is uniquely determined.

Next, observing that $w=0$ along $g_{0}(\bar{\Delta})$ and remembering (10.14), write $(10.18)$ as

$$
0=V_{2}\left(V_{2} r\right) h_{2}+\bar{V}_{2}\left(V_{2} r\right) \bar{h}_{2}+\left(V_{1} r\right) h_{3} \text { on } S^{1}
$$

Introducing

$$
\left(\overline{V_{2} \circ{ }_{0} f}\right)\left(V_{2} \circ{ }_{0} f\right) r=\alpha, \quad\left(V_{2} \circ{ }_{0} f\right)\left(V_{2} \circ{ }_{0} f\right) r=\beta \quad \text { on } S^{1},
$$

(10.28) is rewritten in terms of $\mathrm{H}_{2}, \mathrm{H}_{3}$ as

$$
\alpha \bar{H}_{2}+\beta H_{2}+H_{3} / \zeta+\psi=0 \text { on } S^{1}
$$

where $\psi$ can be expressed through $\varphi_{j}$. (10.16) is equivalent to $H_{3} / \zeta$ being holomorphic even at 0 .

Proposition 10.5. For $\zeta \in S^{1}, \alpha(\zeta)$ is real and $\alpha(\zeta)>|\beta(\zeta)|$.

Accepting this proposition we can apply Lemma 4.2 of [25], which asserts that (10.30) has a unique holomorphic solution $\mathrm{H}_{2}, \mathrm{H}_{3} / \zeta, \frac{3}{2}$-Hölder continuous on $\bar{\Delta}$ such that $H_{2}(1)$ is some given value, say

$$
H_{2}(1)=a_{2}+\varphi_{2}(1)
$$

(as follows from (10.21)). Therefore $H_{1}, H_{2}, H_{3}$ and so $h_{1}, h_{2}, h_{3}$ are uniquely determined by $u_{1}, u_{2}, u_{3}, a_{1}, a_{2}, a_{3}$.

With the functions $h_{1}, h_{2}, h_{3}$, and $h=\sum h_{j} v_{j}$ thus obtained (10.16), (10.17), (10.18), and (10.20) are satisfied, and so is (10.21) for $j=1,2$. It is now straightforward to show that (the second equation of) (10.19) implies that (10.21) is satisfied even for $j=3$. This means that $L$, and therefore $L_{0}=\left(\frac{\partial \Psi}{\partial g}\right)\left(0, g_{0}\right)$, is an isomorphism, whence we get the map $\Phi$ as claimed in the first part of the lemma by solving the equation

$$
\Psi(\Omega, \Phi(\widetilde{p}, \Omega))=(0, \widetilde{p}) .
$$

V. Finally, let us prove that when $\Omega \in U_{1}$ is infinitely differentiable then so is $\Theta$. Observe that, for such an $\Omega, \Psi_{0}(g)=\Psi(\Omega, g)$ smoothly depends on $g$. From (10.31) we obtain

$$
\Psi_{0}(\Theta(\tilde{p}))=(0, \tilde{p}) .
$$

Repeated differentiation of this then gives $\Theta \in C^{\infty}$.

Proof of Proposition 10.5. Let us go back to the definition of $V_{2}$ (see (10.13)). For a fixed $\zeta \in S^{1},\{\mu(\zeta, \omega): \omega \in \mathbb{C}\}=\ell$ is a complex, affine line in $\mathbb{C}^{2}$, tangent to $M_{0}=\partial D_{0}$ in $\mu(\zeta, 0)$. Strict linear convexity implies that $\ell$ entirely lies in $\bar{D}_{0}$, and more precisely, the function $\rho(\omega)=r(\mu(\zeta, \omega))$ attains a 
nondegenerate minimum at $\omega=0$. Hence $\rho_{\omega \bar{\omega}}(0)>\left|\rho_{\omega \omega}(0)\right|$, so that in view of (10.13) and (10.29) we obtain the statement of the proposition.

An easy consequence of the proof of Lemma 10.3 is the following

Proposition 10.6. Let $p(t)$ be a smooth curve on $M, p(0)=p$. Then the vector field

$$
Y=\left.\frac{d}{d t}\right|_{t=0} f^{p(t)}
$$

along ${ }_{0} f^{p}$ is either everywhere tangent or everywhere transverse to ${ }_{0} f^{p}(\bar{\Delta})$.

Proof. With liftings $g_{0}^{p(t)}: \bar{\Delta} \rightarrow \bar{Q}$ of ${ }_{0} f^{p(t)}$ we have $\Psi\left(0, g_{0}^{p(t t)}\right)=(0, p(t))$. It follows that the section $h$ of $g_{0}^{p *} T^{1,0} \bar{Q}$ defined by $\operatorname{Re} h=\left(\frac{d}{d t}\right)_{t=0} g_{0}^{p(t)}$ satisfies (10.9), (10.10), and $\bar{\partial} h=0$. Furthermore, the $(1,0)$ component of $Y$ is

$$
\pi^{1,0} Y=\left.\pi^{1,0} \Pi_{*} \frac{d}{d t}\right|_{t=0} g_{0}^{p(t)}=\frac{1}{2} \Pi_{*} h=\frac{1}{2} \sum_{j=1}^{2} h_{j} V_{j}\left({ }_{0} f^{p}\right) .
$$

Hence $Y(\zeta)$ is transverse to ${ }_{0} f^{p}(\bar{\Delta})$ if and only if $h_{2}(\zeta) \neq 0$.

In the computations that lead to the inversion of $L$ in this case we can now choose $\varphi_{j}=0$, whence $H_{j}=h_{j}$ and $\psi=0$. This means that (10.30) has the trivial solution $H_{2} \equiv H_{3} \equiv 0$. But Lemma 4.2 of [25] asserts that for any $\zeta_{0} \in \bar{\Delta}(10.30)$ is satisfied by a unique couple $H_{2}, H_{3} / \zeta$ of holomorphic functions such that $H_{2}\left(\zeta_{0}\right)=0$. Thus, if $H_{2}=h_{2}$ vanishes somewhere, it vanishes everywhere. This proves our claim.

There is an obvious $S^{1}$ action on the manifold Map given for $\omega \in S^{1}$ by $g(\zeta) \mapsto g(\omega \zeta)(g \in \mathrm{Map})$, and it can be assumed that $U_{2}$ in Lemma 10.3 is invariant under this action ( $K$ certainly is). Also, for any fixed $\Omega \in U_{1}$ the set of $\Omega$-holomorphic $g \in$ Map is also invariant. This implies the following symmetry property of $\Phi$ :

Proposition 10.7. For some $\Omega \in U_{1}, \tilde{p} \in \widetilde{M}_{0}$ let $g=\Phi(\Omega, \tilde{p}) \in U_{2}$, and for some $\omega \in S^{1}$, put $G(\zeta)=g(\zeta \omega), \widetilde{P}=G(1)=g(\omega)$. Then $G=\Phi(\Omega, \widetilde{P})$.

Proof of Theorem 10.1. There is a smooth $\mathbb{C}$-linear bundle projection

$$
\mathbb{C} \otimes T \bar{D}=\mathbb{C} \otimes T \bar{D}_{0} \rightarrow T^{1,0} \bar{D}_{0}
$$

which restricts to an isomorphism $T^{1,0} \bar{D} \rightarrow T^{1,0} \bar{D}_{0}$. This isomorphism, in turn, defines a diffeomorphism

$$
\delta: \mathbb{P}^{*}\left(T^{1,0} \bar{D}\right) \rightarrow \mathbb{P}^{*}\left(T^{1,0} \bar{D}_{0}\right)=\bar{Q} .
$$

Pushing forward the complex structure of $\mathbb{P}^{*}\left(T^{1,0} \bar{D}\right)$ we obtain a new complex structure $\bar{Q}_{\Omega}$ on $\bar{Q}$ as above. One checks that if the complex structures of $\bar{D}, \bar{D}_{0}$ are sufficiently $C^{b+1}$-close, then $\Omega \in \operatorname{Str}_{b}$ will be as close to 0 as we need. Also, $\Omega$ is smooth. Further, with

$$
\widetilde{M}=\left\{H_{p}^{1,0} M: p \in M\right\}, \quad C=\left\{T_{p}^{1,0} \mathbb{P}_{1}(\infty): p \in M\right\},
$$


we have $\delta(\widetilde{M})=\widetilde{M}_{0}$ and $\delta(C)=C_{0}$. For $\widetilde{M}$ this follows from $H M=$ $H M_{0}$ while for $C$ actually a stronger assertion holds: since, for points $p \in$ $\mathbb{P}_{1}(\infty), T_{p}^{1,0} D$ and $T_{p}^{1,0} D_{0}$ coincide, $C=C_{0}$, and the diffeomorphism $\delta$ fixes points in $C$. Observe also that the projection $\Pi: Q \rightarrow D_{0}$ is holomorphic when viewed as a projection $Q_{\Omega} \rightarrow D$.

Now let $p \in M$. Put $\widetilde{p}=\delta\left(H_{p}^{1,0} M\right)=H_{p}^{1,0} M_{0}$ and with the mapping $\Phi$ of Lemma 10.3

$$
\begin{aligned}
& g^{p}=\Phi(\Omega, \widetilde{p}): \bar{\Delta} \rightarrow \bar{Q}_{\Omega}, \\
& f^{p}=\Pi \circ g^{p}: \bar{\Delta} \rightarrow \bar{D}, \\
& E_{\zeta}^{p}=\delta^{-1}\left(g^{p}(\zeta)\right) \in \mathbb{P}^{*}\left(T_{f^{p}(\zeta)}^{1,0} \bar{D}\right) .
\end{aligned}
$$

Then $f^{p}$ is holomorphic from $\Delta$ to $D$ and $\left\{E_{\zeta}^{p}: \zeta \in \Delta\right\}$ defines a holomorphic line subbundle $E^{p}$ of $f^{p *} T^{1,0} \bar{D}$. However, a priori these objects are only $\frac{3}{2}$ Hölder continuous on $\bar{D}$.

Proposition 10.8. $g^{p} \in C^{\infty}(\bar{\Delta})$; hence $f^{p}$ and $E^{p}$ are also smooth on $\bar{\Delta}$. In addition, $g^{p}(\zeta)$ depends smoothly on $(p, \zeta) \in M \times \bar{\Delta}$.

Proof. Since $\Omega$ is smooth, $g^{p} \in$ Map depends smoothly on $p \in M$, and so

$$
V:\left.\widetilde{M}_{0} \ni \widetilde{p} \mapsto \frac{d}{d t}\right|_{t=0} g^{\Pi \widetilde{p}}\left(e^{i t}\right) \in T_{\widetilde{p}} \widetilde{M}_{0}
$$

defines a smooth vector field on $\widetilde{M}_{0}$. In view of Proposition 10.7 the curves $t \mapsto g^{p}\left(e^{i t}\right)$ are trajectories of this vector field, and hence they are smooth. In fact, the entire dynamics $(p, t) \mapsto g^{p}\left(e^{i t}\right)$ is smooth. Since $g=g^{p}$ are $\Omega$-holomorphic, i.e., solutions of (10.4), and their boundary values depend smoothly on $p$, the simplest instance of elliptic regularity theory now implies that $g^{p}(\zeta)$ depends smoothly on $(p, \zeta) \in M \times \bar{\Delta}$.

We can now check that $f=f^{p}, E=E^{p}$ have the properties listed in Theorem 10.1. (a),(b), and (d) follow from properties of $\Phi$; (e) from Proposition 10.7. Transversality is a stable concept; (c) holds for $\Omega=0$, and therefore it holds for small $\Omega$. By the same reason the maps $f^{p}$ are embeddings.

As to (f), Proposition 10.8 implies that

$$
F(p, \zeta)=\Pi g^{p}(\zeta)
$$

is smooth. To see that $F\left(\cdot, \zeta_{0}\right)$ is an immersion $\left(\zeta_{0} \neq 0\right)$ for every $p \in M$ we have to exhibit three vectors in $T_{p} M$ such that their images under $F_{*}\left(\cdot, \zeta_{0}\right)$ are independent. One of the vectors will be

$$
\left.\frac{d}{d t}\right|_{t=0} F\left(p, e^{i t}\right)=\left.\frac{d}{d t}\right|_{t=0} f^{p}\left(e^{i t}\right) .
$$

Its image is $\left(d f^{p}\left(\zeta_{0} e^{i t}\right) / d t\right)_{t=0} \neq 0$, a vector tangent to $f^{p}(\bar{\Delta})$. Next choose two orthonormal vectors $\eta_{1}, \eta_{2} \in H_{p} M$. Let $p_{j}(t)(j=1,2)$ be curves in $M$ such that $p_{j}(0)=p,\left(d p_{j}(t) / d t\right)_{t=0}=\eta_{j}$. Then the image of $\eta_{j}$ under $F_{*}\left(\cdot, \zeta_{0}\right)$ is 
$Y_{j}\left(\zeta_{0}\right)$ with $Y_{j}$ given by

$$
Y_{j}(\zeta)=\left.\frac{d}{d t}\right|_{t=0} f^{p_{j}(t)}(\zeta)
$$

It suffices to prove that, for $c_{1}, c_{2} \in \mathbb{R} \backslash\{0\}, c_{1} Y_{1}\left(\zeta_{0}\right)+c_{2} Y_{2}\left(\zeta_{0}\right)$ is transverse to $f^{p}(\bar{\Delta})$. Actually this can be proved for any $\zeta_{0} \in \bar{\Delta}$. By Proposition 10.6 it is true when $D=D_{0}$ (observe $Y_{j}(1)=\eta_{j}$ ). Therefore it is true for a slightly perturbed situation, too. (At that point it is essential that $\zeta_{0}$ can range over the compact set $\bar{\Delta}$ rather than $\bar{\Delta} \backslash\{0\}$; and, of course, one can also restrict $c_{1}, c_{2}$ to the compact set $\left\{c_{1}^{2}+c_{2}^{2}=1\right\}$.)

11. Geometric considerations. We continue to study the structure of a small perturbation $M$ of a strictly linearly convex hypersurface $M_{0} \subset \mathbb{C}^{2} \subset \mathbb{P}_{2}$. In the previous section we constructed a free outer $S^{1}$ action $\left\{g_{\zeta}\right\}$ on $M$. In fact this outer action is a small perturbation of the outer action $\left\{g_{\zeta}^{0}\right\}$ on $M_{0}$ constructed in $\S 8$. The latter action is differentiably conjugate to the standard $S^{1}$ action $z \mapsto \zeta z \quad\left(\zeta \in S^{1}\right)$ on a strictly convex circular hypersurface as explained at the end of $\S 8$, and thus exhibits $M_{0}$ as a principal $S^{1}$ bundle over $\mathbb{P}_{1}$. Such bundles are classified by their (first) Chern class; ours has Chern class 1. The same holds therefore for $M$.

Lemma 11.1. There is a contact diffeomorphism $\gamma: M_{0} \rightarrow M$ which intertwines the actions $\left\{g_{\zeta}\right\}$ and $\left\{g_{\zeta}^{0}\right\} . \gamma$ will be as near to the identity as we please if $\left\{g_{\zeta}\right\}$ is a sufficiently small perturbation of $\left\{g_{\zeta}^{0}\right\}$.

Proof. The $\left\{g_{\zeta}\right\}$ invariant contact structure can be regarded as a connection on the principal bundle $M \rightarrow \mathbb{P}_{1}$. The curvature of this connection is a smooth 2-form $\omega$ on $\mathbb{P}_{1}$, which is everywhere positive in view of the strict pseudoconvexity of $M$. Also $\int_{\mathbb{P}_{1}} \omega=4 \pi$, since $\omega / 4 \pi$ is the Chern form.

Similarly, we have a connection on $M_{0} \rightarrow \mathbb{P}_{1}$ with curvature form $\omega_{0}>$ $0, \int_{\mathbb{P}_{1}} \omega_{0}=4 \pi$. This implies that there is a diffeomorphism $\gamma_{0}: \mathbb{P}_{1} \rightarrow \mathbb{P}_{1}$ such that $\gamma_{0}^{*} \omega=\omega_{0}$ (see [28]). The induced bundle $\gamma_{0}^{*} M=M^{\prime}$ (with connection) is of course isomorphic to $M$, and the curvature of $M^{\prime} \rightarrow \mathbb{P}_{1}$ is $\omega_{0}$. Since $\mathbb{P}_{1}$ is simply connected, this implies that there is a connection-preserving diffeomorphism $f: M_{0} \rightarrow M^{\prime}$ which makes the diagram

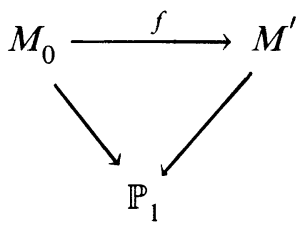

commutative. Composing $f$ with the isomorphism $M^{\prime} \rightarrow M$ we obtain $\gamma$ as required. In fact, if the actions $\left\{g_{\zeta}\right\},\left\{g_{\zeta}^{0}\right\}$ are $C^{a}$ near, the diffeomorphism $\gamma$ will be $C^{a-3}$ near the identity (one loses two derivatives at the computation of the curvature and then another one at the construction of $\gamma$ ). 
An equivalent way of stating this lemma is that any small perturbation of $M_{0}$ is equivalent to one for which the action $\left\{g_{\zeta}^{0}\right\}$ is an outer action. In what follows, we shall assume that $M$ is such a perturbation. Thus $g_{\zeta}=g_{\zeta}^{0}$.

Given an outer $S^{1}$ action on $M$ (or $M_{0}$ ), the proof of Theorem 6.5, or rather Theorem 4.1, mutatus mutandis, constructs in a canonical way a strictly pseudoconcave surface $\bar{E}$ (resp. $\bar{E}_{0}$ ) whose boundary is $M$ (resp. $M_{0}$ ). The smooth structure of $\bar{E}$ depends only on the $S^{1}$ action but not the CR structure on $M$. Hence the underlying differentiable manifolds of $\bar{E}$ and $\bar{E}_{0}$ coincide. Also parts of the complex structures in $\bar{E}$ and $\bar{E}_{0}$ coincide.

More precisely, observe that (differentiably) $\bar{E}_{0}$ is constructed as a disc bundle over $\mathbb{P}_{1}=M_{0} / S^{1}$, the fibers are complex submanifolds and $\bar{\Delta}^{\prime}=\mathbb{P}_{1} \backslash \Delta$ acts holomorphically on them. $\bar{E} \rightarrow \mathbb{P}_{1}$ of course has the same fibers and the same complex structure on the fibers. (This reflects the fact that the $(0,1)$ vector field $\bar{W}$ in the proof of Theorem 4.1 does not depend on the Beltrami differentials, i.e., the CR structure of $M_{0}$, resp. $M$.) The fixed point sets of the $\bar{\Delta}^{\prime}$ action on $\bar{E}$ and $\bar{E}_{0}$ are, of course, the same and are complex submanifolds both in $\bar{E}$ and $\bar{E}_{0}$ but their complex structures in general will be different (although, of course, isomorphic to the Riemann sphere $\mathbb{P}_{1}$ ). We will denote these fixed sets by $C$, resp. $C_{0}$.

According to Lemma 7.1, and properties of the exterior circular representation explained at the end of $\S 8, E_{0}$ is biholomorphic to the pseudoconcave component $D_{0}$ of $\mathbb{P}_{2} \backslash M$. Also $E$ is biholomorphic to $D$ of $\S 10$, but we shall not need this. We shall, however, need the fact that the normal bundle of $C_{0}$ in $E_{0}$, resp. $C$, in $E$ has Chern class 1, a fact equivalent to the corresponding property of the $S^{1}$ bundles $M_{0} \rightarrow \mathbb{P}_{1}$, resp. $M \rightarrow \mathbb{P}_{1}$.

We will also need a vanishing theorem for $\bar{\partial}_{b}$ cohomology on $M_{0}$ or $M$. Endow, say, $M$ with a smooth, $\left\{g_{\zeta}\right\}$ invariant volume form, and define the Hilbert space $L^{2}(M)$ of square integrable functions on $M$ using this volume form. Then the $S^{1}$ action $\left\{g_{\zeta}\right\}$ decomposes $L^{2}(M)$ into orthogonal eigenspaces $L_{k}^{2}(M), k=0, \pm 1, \ldots$ :

$$
L_{k}^{2}(M)=\left\{\varphi \in L^{2}(M): \varphi \circ g_{\zeta}=\zeta^{k} \varphi, \zeta \in S^{1}\right\} .
$$

Put $L_{-}^{2}(M)=\widehat{\bigoplus}_{k \leq 1} L_{k}^{2} M$. Thus $\varphi \in L^{2} M$ is in $L_{-}^{2} M$ if and only if $\varphi$ has an extension to $\bar{E} \backslash C$ which is holomorphic along the fibers of $E \rightarrow \mathbb{P}_{1}$ (orbits of the $\Delta^{\prime}$ action) and has at most first-order poles in points of $C$. Introducing the line bundle $[C]$ on $\bar{E}$ determined by the divisor $C$ and a holomorphic section $\sigma$ of $[C]$, whose divisor is $C$, we can also say that $\varphi \in L^{2}(M)$ is in $L_{-}^{2}(M)$ if $\varphi \sigma$ has an extension to a section $\tilde{\varphi}$ of $[C]$, holomorphic along the fibers of $E \rightarrow \mathbb{P}_{1}$.

Given now a continuous $\varphi \in L_{-}^{2}(M)$ we obtain a continuous section $\tilde{\varphi}$ of $[C]$ as above. Using some hermitian metrics on $C$ and $\left.[C]\right|_{C}$, project $\left.\tilde{\varphi}\right|_{C}$ on the space of holomorphic sections of $\left.[C]\right|_{C}$. Denote the projection $\omega_{1}(\varphi) \epsilon$ 
$H^{0}\left(\left.[C]\right|_{C}\right)$. Also put $\omega_{2}(\varphi)=\int_{M} \varphi \in \mathbb{C}$. (This could also be interpreted as a cohomology class but we shall skip this point.) Then $\omega(\varphi)=\left(\omega_{1}(\varphi), \omega_{2}(\varphi)\right)$ measures in some sense the component of $\varphi$ in $L_{1}^{2}(M) \oplus L_{0}^{2}(M)$.

Theorem 11.1. If $\varphi \in C^{1}(M) \cap L_{-}^{2}(M) \cap \operatorname{Ker} \omega$ is a CR function then $\varphi=0$.

Proof. With $\widetilde{\varphi}$ as above we first show that $\widetilde{\varphi}$ is holomorphic. It suffices to show this off $C$, where $\widetilde{\varphi} \sigma^{-1}=\widetilde{\widetilde{\varphi}}$ is a $C^{1}$ function, $\left.\widetilde{\widetilde{\varphi}}\right|_{M}=\varphi$. Let $X$ be a $\bar{\Delta}^{\prime}$ invariant field of $(1,0)$-like vectors along an orbit $O$ of the action $\left\{g_{\zeta}: \zeta \in \bar{\Delta}^{\prime}\right\}$ on $E$, which, in points of $O \cap M$, is tangent to $M_{0}$. (Cf. the proof of Theorem 4.1 , with $\bar{\Delta}$ replaced by $\bar{\Delta}^{\prime}, \bar{D}$ by $\bar{E}$. Also notice that since the $S^{1}$ action on $M$ is free, there is no need to factor by a finite group, so $\widetilde{E}_{B}=\bar{E}_{B}$ and $h_{\zeta}=g_{\zeta}$.) If $\mu(\zeta)$ is the Beltrami differential associated with $X_{p}$ ( $p$ a point on the $\bar{\Delta}^{\prime}$ orbit $O$ ), then

$$
\bar{Z}_{g_{\zeta}(p)}=\bar{X}_{g_{\zeta}(p)}+\mu(\zeta) X_{g_{\zeta}(p)} \quad\left(\zeta \in \bar{\Delta}^{\prime}\right)
$$

is a $(0,1)$ vector field, cf. (5.8).

Since $\widetilde{\widetilde{\varphi}}$ is holomorphic along orbits and $X, \bar{X}$ are invariant, $\bar{X}_{g_{\zeta}(p)} \widetilde{\widetilde{\varphi}}$ and $X_{g_{\zeta}(p)} \widetilde{\widetilde{\varphi}}$ are holomorphic functions of $\zeta \in \Delta^{\prime} \backslash\{0\}$, continuous for $\zeta \in \bar{\Delta}^{\prime} \backslash\{0\}$. The same holds for $\bar{Z}_{g_{\zeta}(p)} \approx \widetilde{\tilde{\varphi}}$. Now this latter vanishes for $\zeta \in S^{1}$ because $\varphi$ is $\mathrm{CR}$ and $\left.\bar{Z}\right|_{M}$ is tangential to $M$, so it is identically zero. From this it follows that $\widetilde{\tilde{\varphi}}$, and so $\widetilde{\varphi}$, is holomorphic.

Thus $\left.\widetilde{\varphi}\right|_{C}$ is a holomorphic section of $\left.[C]\right|_{C}$. By the assumption $\omega_{1}(\widetilde{\varphi})=0$ we have $\left.\widetilde{\varphi}\right|_{C}=0$. So $\widetilde{\widetilde{\varphi}}$ is a holomorphic function on the whole of $\bar{E}$. The boundary $M$ of $\bar{E}$ is strictly pseudoconcave. By shrinking $\bar{E}$ a little we can construct a pseudoconcave subdomain $F, \bar{F} \subset E$. $|\tilde{\tilde{\varphi}}|$ has to attain its maximum on $\bar{F}$ in a point $q \in \partial F$. However, because of strict pseudoconcavity, there is a holomorphic disc $\delta \subset \bar{F}$ such that $\delta \cap \partial F=\{q\}$, so that the maximum principle implies $\left.\widetilde{\tilde{\varphi}}\right|_{\delta}=$ const. Hence $|\widetilde{\tilde{\varphi}}|$ attains its maximum also in interior points of $\bar{F}$, whence $\tilde{\tilde{\varphi}}$ is constant, and therefore so is $\varphi$. Because $\omega_{2}(\varphi)=\int_{M} \varphi=0$, this constant has to be 0 .

12. Analytic considerations. Let $M,\left\{g_{\zeta}\right\}$ be as in $\S 11$. We want to prove precise estimates for the boundary operator $\bar{\partial}_{b}$ acting on $L_{-}^{2}(M)$. The appropriate norms for precise estimates are the Folland-Stein norms. These are defined as follows (see [10]). Let $Y_{1}, \ldots, Y_{\nu}$ be smooth sections of $H M$ that span $H M$ in every point. We can assume they are $\left\{g_{\zeta}\right\}$ invariant. Put $Y^{N}=Y_{\nu_{1}} Y_{\nu_{2}} \cdots Y_{\nu_{j}}$ if $N$ is the multiindex $\left(\nu_{1}, \ldots, \nu_{j}\right)$. In this case also put $|N|=j$. The Folland-Stein norms \|\|$_{s}$ on $C^{\infty}(M)$ are given by

$$
\|\Phi\|_{s}^{2}=\sum_{|N| \leq s}\left\|Y^{N} \Phi\right\|^{2}, \quad s=0,1,2, \ldots
$$


where \|\|$=\|\|_{0}$ is the $\left(\left\{g_{\zeta}\right\}\right.$ invariant $) L^{2}$ norm. Also $\langle$,$\rangle will$ denote the inner product on $L^{2}(M)$.

Let us further choose smooth sections $\bar{Z}_{1}, \ldots, \bar{Z}_{\nu}$ of $H^{0,1}(M)$ that span $H^{0,1}(M)$ in every point. They cannot, in general, be chosen $\left\{g_{\zeta}\right\}$ invariant, and so will not preserve the grading $L_{k}^{2}(M)$, but this will be of no importance to us.

For $\Phi \in C^{\infty}(M)$ we shall represent $\bar{\partial}_{b} \Phi$ by $\left\{\bar{Z}_{\alpha} \Phi: 1 \leq \alpha \leq \nu\right\}$. In particular, we put

$$
\left\|\bar{\partial}_{b} \Phi\right\|_{s}^{2}=\sum_{\alpha=1}^{\nu}\left\|\bar{Z}_{\alpha} \Phi\right\|_{s}^{2}
$$

Let us introduce the vector field $V=\left(d g_{e^{i t}} / d t\right)_{t=0}$, the infinitesimal generator of the $S^{1}$ action. If $\Phi \in L_{k}^{2} M, V \Phi=i k \Phi$ so that $i V$ is bounded from below on $L_{-}^{2} M$.

We have the following lemma, a cousin of Theorem 15.3 in [4].

Lemma 12.1. There are constants $C_{s}$ such that for $\Phi \in L_{-}^{2}(M) \cap C^{\infty}(M)$

$$
\begin{aligned}
& \|\Phi\|_{s} \leq C_{s}\left(\left\|\bar{\partial}_{b} \Phi\right\|_{s-1}+\|\Phi\|_{0}\right), \\
& \|V \Phi\|_{s} \leq C_{s}\left(\left\|\bar{\partial}_{b} \Phi\right\|_{s+1}+\|\Phi\|_{0}\right)
\end{aligned}
$$

for $s=1,3,5 \ldots$.

For brevity introduce the notation $a \prec b$ to mean that there is some constant $C$ (possibly depending on $s$, but not on the particular function $\Phi$ involved in $a$ and $b$ ) such that $a \leq C b$.

Proposition 12.2. (12.1) holds for $s=1$ : if $\Phi \in L_{-}^{2}(M) \cap C^{\infty}(M)$ then

$$
\|\Phi\|_{1} \prec\left\|\bar{\partial}_{b} \Phi\right\|_{0}+\|\Phi\|_{0}
$$

Proof. By pseudoconvexity (cf. (5.15), (5.16))

$$
\sum_{\alpha}\left[\bar{Z}_{\alpha}, Z_{\alpha}\right]=i p V+\sum_{\alpha}\left(c_{\alpha} Z_{\alpha}+c_{\alpha}^{\prime} \bar{Z}_{\alpha}\right),
$$

where $p$ is an everywhere positive smooth function on $M$. Replacing $Z_{\alpha}$ by $p^{-1 / 2} Z_{\alpha}$ we can achieve that in (12.4) $p=1$.

Since the adjoint of $Z_{\alpha}$ is $-\bar{Z}_{\alpha}+c$ with $c$ a smooth function, we have

$$
\begin{aligned}
\sum_{\alpha}\left\langle Z_{\alpha} \Phi, Z_{\alpha} \Phi\right\rangle & =-\sum_{\alpha}\left\langle\bar{Z}_{\alpha} Z_{\alpha} \Phi, \Phi\right\rangle+O\left(\|\Phi\|_{0}\|\Phi\|_{1}\right) \\
& =-\sum_{\alpha}\left\langle Z_{\alpha} \bar{Z}_{\alpha} \Phi, \Phi\right\rangle-\sum_{\alpha}\left\langle\left[\bar{Z}_{\alpha}, Z_{\alpha}\right] \Phi, \Phi\right\rangle+O\left(\|\Phi\|_{0}\|\Phi\|_{1}\right) \\
& =\sum_{\alpha}\left(\bar{Z}_{\alpha} \Phi, \bar{Z}_{\alpha} \Phi\right\rangle-\langle i V \Phi, \Phi\rangle+O\left(\|\Phi\|_{0}\|\Phi\|_{1}\right) .
\end{aligned}
$$


Since $\langle i V \Phi, \Phi\rangle+\|\Phi\|_{0}^{2} \geq 0$ on $L_{-}^{2}(M)$, we obtain

$$
\begin{aligned}
\|\Phi\|_{1}^{2} & \prec \sum_{\alpha}\left\langle Z_{\alpha} \Phi, Z_{\alpha} \Phi\right\rangle+\sum_{\alpha}\left\langle\bar{Z}_{\alpha} \Phi, \bar{Z}_{\alpha} \Phi\right\rangle+\|\Phi\|_{0}^{2} \\
& \prec \sum_{\alpha}\left\langle\bar{Z}_{\alpha} \Phi, \bar{Z}_{\alpha} \Phi\right\rangle+\|\Phi\|_{0}\|\Phi\|_{1} \\
& \prec\left\|\bar{\partial}_{b} \Phi\right\|_{0}^{2}+\|\Phi\|_{0}\|\Phi\|_{1} .
\end{aligned}
$$

From this (12.3) follows.

Proposition 12.3. If $\Phi, \Psi \in C^{\infty}(M)$ then

$$
|\langle V \Phi, \Psi\rangle| \prec\|\Phi\|_{1}\|\Psi\|_{1} .
$$

Proof.

$$
\begin{aligned}
|\langle i V \Phi, \Psi\rangle| & =\left|\sum_{\alpha}\left\langle\left[\bar{Z}_{\alpha}, Z_{\alpha}\right] \Phi, \Psi\right\rangle\right|+O\left(\|\Phi\|_{1}\|\Psi\|_{0}\right) \\
& \prec \sum_{\alpha}\left|\left\langle\bar{Z}_{\alpha} \Phi, \bar{Z}_{\alpha} \Psi\right\rangle\right|+\sum_{\alpha}\left|\left\langle Z_{\alpha} \Phi, Z_{\alpha} \Psi\right\rangle\right|+O\left(\|\Phi\|_{1}\|\Psi\|_{1}\right) \\
& \prec\|\Phi\|_{1}\|\Psi\|_{1} .
\end{aligned}
$$

Remark 12.4. For $\Phi \in L_{-}^{2}(M)$, combining this result with Proposition 12.2 we obtain (12.2) with $s=-1$.

Proposition 12.5. (12.2) holds for $s=1$.

Proof. In (12.3) replace $\Phi$ by $V \Phi$ :

$$
\|V \Phi\|_{1}^{2} \prec \sum_{\alpha}\left\|\bar{Z}_{\alpha} V \Phi\right\|_{0}^{2}+\|V \Phi\|_{0}^{2} .
$$

By (12.6) we have

$$
\|V \Phi\|_{0}^{2} \prec\|\Phi\|_{1}\|V \Phi\|_{1}
$$

Also

$$
\left\langle\bar{Z}_{\alpha} V \Phi, \bar{Z}_{\alpha} V \Phi\right\rangle=\left\langle V \bar{Z}_{\alpha} \Phi, \bar{Z}_{\alpha} V \Phi\right\rangle+O\left(\|\Phi\|_{1}\|V \Phi\|_{1}\right)
$$

because $\left\{g_{\zeta}\right\}$ preserves the contact structure so that $\left[V, \bar{Z}_{\alpha}\right]$ is a combination of $Y_{\beta}$. Next, the adjoint of $\bar{Z}_{\alpha}$ being $-Z_{\alpha}+\bar{c}, c$ a smooth function

$$
\begin{aligned}
& \left\langle V \bar{Z}_{\alpha} \Phi, \bar{Z}_{\alpha} V \Phi\right\rangle=-\left\langle Z_{\alpha} V \bar{Z}_{\alpha} \Phi, V \Phi\right\rangle+\left\langle V \bar{Z}_{\alpha} \Phi, \bar{c} V \Phi\right\rangle \\
& \quad=-\left\langle V Z_{\alpha} \bar{Z}_{\alpha} \Phi, V \Phi\right\rangle+O\left(\left\|\bar{\partial}_{b} \Phi\right\|_{1}\|V \Phi\|_{0}\right)+O\left(\left\|\bar{\partial}_{b} \Phi\right\|_{1}\|V \Phi\|_{1}\right),
\end{aligned}
$$

where we again used $\left[V, Z_{\alpha}\right]=\sum c_{\beta} Y_{\beta}$, and (12.6) The first term here is, again by (12.6) $O\left(\left\|\bar{\partial}_{b} \Phi\right\|_{2}\|V \Phi\|_{1}\right)$, so that finally from (12.7) we get

$$
\|V \Phi\|_{1}^{2} \prec\left\|\bar{\partial}_{b} \Phi\right\|_{2}\|V \Phi\|_{1}+\|\Phi\|_{1}\|V \Phi\|_{1} .
$$

Dividing by $\|V \Phi\|_{1}$ and substituting (12.3) we obtain (12.2) with $s=1$. 
Proof of Lemma 12.1. We shall prove by induction. For $s=1$ both estimates are proved. Assuming for some $s$ (12.1) and (12.2) hold, we can estimate

$$
\begin{aligned}
\|\Phi\|_{s+2}^{2} & =\sum_{\alpha, \beta}\left\|Y_{\alpha} Y_{\beta} \Phi\right\|_{s}^{2}+\|\Phi\|_{1}^{2} \\
& \prec \sum_{\alpha, \beta}\left\|\bar{\partial}_{b}\left(Y_{\alpha} Y_{\beta} \Phi\right)\right\|_{s-1}^{2}+\left\|Y_{\alpha} Y_{\beta} \Phi\right\|_{0}^{2}+\|\Phi\|_{1}^{2} \\
& \prec \sum_{\alpha, \beta, \gamma}\left\|\bar{Z}_{\gamma} Y_{\alpha} Y_{\beta} \Phi\right\|_{s-1}^{2}+\|\Phi\|_{2}^{2} .
\end{aligned}
$$

As before, since $\left[\bar{Z}_{\gamma}, Y_{\alpha}\right]=c V+\sum c_{\delta} Y_{\delta}$, we have

$$
\begin{aligned}
\left\|\bar{Z}_{\gamma} Y_{\alpha} Y_{\beta} \Phi\right\|_{s-1}^{2} & \prec\left\|Y_{\alpha} \bar{Z}_{\gamma} Y_{\beta} \Phi\right\|_{s-1}^{2}+\left\|V Y_{\beta} \Phi\right\|_{s-1}^{2}+\|\Phi\|_{s+1}^{2} \\
& \prec\left\|Y_{\alpha} Y_{\beta} \bar{Z}_{\gamma} \Phi\right\|_{s-1}^{2}+\left\|Y_{\alpha} V \Phi\right\|_{s-1}^{2}+\left\|Y_{\beta} V \Phi\right\|_{s-1}^{2}+\|\Phi\|_{s+1}^{2} \\
& \prec\left\|\bar{\partial}_{b} \Phi\right\|_{s+1}^{2}+\|V \Phi\|_{s}^{2}+\|\Phi\|_{s+1}^{2} \\
& \prec\left\|\bar{\partial}_{b} \Phi\right\|_{s+1}^{2}+\|\Phi\|_{s+1}^{2} .
\end{aligned}
$$

Thus, from (12.8)

$$
\|\Phi\|_{s+2} \prec\left\|\bar{\partial}_{b} \Phi\right\|_{s+1}+\|\Phi\|_{s+1} .
$$

From this one proves

$$
\|\Phi\|_{s+2} \prec\left\|\bar{\partial}_{b} \Phi\right\|_{s+1}+\|\Phi\|_{0}
$$

indirectly. If (12.10) were not true, there would be a sequence $\Phi_{k} \in L_{-}^{2}(M) \cap$ $C^{\infty}(M)$ such that $\left\|\Phi_{k}\right\|_{s+2}=1$ but $\left\|\bar{\partial}_{b} \Phi_{k}\right\|_{s+1}+\left\|\Phi_{k}\right\|_{0} \rightarrow 0$. We could select a subsequence (still denoted $\Phi_{k}$ ) which converges in \|\|$_{s+1}$. Of course, the limit has to be 0 ; i.e., $\left\|\Phi_{k}\right\|_{s+1} \rightarrow 0$, contradicting (12.9). Thus (12.1) is proved with $s$ replaced by $s+2$.

Next, using (12.10) with $\Phi$ replaced by $V \Phi$

$$
\begin{aligned}
\|V \Phi\|_{s+2} & \prec\left\|\bar{\partial}_{b} V \Phi\right\|_{s+1}+\|V \Phi\|_{0} \\
& \prec \sum_{\alpha}\left\|\bar{Z}_{\alpha} V \Phi\right\|_{s+1}+\|\Phi\|_{2} \\
& \prec \sum_{\alpha}\left\|V \bar{Z}_{\alpha} \Phi\right\|_{s+1}+\|\Phi\|_{s+2} \\
& \prec\left\|\bar{\partial}_{b} \Phi\right\|_{s+3}+\|\Phi\|_{0} .
\end{aligned}
$$

Therefore (12.2) is proved with $s$ replaced by $s+2$, and the induction is complete.

Theorem 12.6. If $s \geq 7$ is odd, and $\Phi \in L_{-}^{2}(M) \cap C^{\infty}(M) \cap \operatorname{Ker} \omega$ then

$$
\|\Phi\|_{s} \prec\left\|\bar{\partial}_{b} \Phi\right\|_{s-1} \text {. }
$$

Proof. Assume this is not true. Since by Lemma 12.1 the left-hand side is dominated by $\left\|\bar{\partial}_{b} \Phi\right\|_{s-1}+\|\Phi\|_{0}$, it follows that there is a sequence $\Phi_{k} \in$ $L_{-}^{2}(M) \cap C^{\infty}(M)$ such that

$$
\left\|\Phi_{k}\right\|_{0}=1, \quad\left\|\bar{\partial}_{b} \Phi_{k}\right\|_{s-1} \rightarrow 0
$$


Lemma 12.1 implies that $\left\|\Phi_{k}\right\|_{s}$ is a bounded sequence, so that a subsequence $\left\{\varphi_{k}\right\}$ is convergent in the \|\|$_{s-1}$ norm. By the embedding theorem of [10] $\varphi_{k}$ converges in $C^{1}(M)$ to a function $\varphi \in L_{-}^{2}(M) \cap C^{1}(M)$. Then $\omega(\varphi)=0$ and $\bar{\partial}_{b} \varphi=0$, so that by Theorem $11.2 \varphi \equiv 0$. This of course contradicts (12.12).

A minor modification of the proof would also give the theorem for $s=$ $1,3,5$.

13. The stability of embeddings. We are now ready to prove Theorem 4.5. Let $M_{0} \subset \mathbb{C}^{2}$ be as there, and let $\left\{g_{\zeta}\right\}$ denote an outer $S^{1}$ action on $M_{0}$. Let $M$ be a small perturbation of $M_{0}$. By Lemma 11.1 we can assume that $\left\{g_{\zeta}\right\}$ is an outer action on $M$ as well. Let $\bar{E}_{0}, \bar{E}$ be the pseudoconcave surfaces constructed in the proof of Theorem 6.5 for $M_{0}$, resp. $M$. They are disc bundles over $\mathbb{P}_{1}$; in fact the smooth structures of $\bar{E}_{0} \rightarrow \mathbb{P}_{1}, \bar{E} \rightarrow \mathbb{P}_{1}$ agree. The zero sections (or, perhaps one should say: $\infty$ sections) are complex curves denoted $C_{0}, C$. As sets they agree but their complex structures are different, although both are biholomorphic to $\mathbb{P}_{1}$. Their normal bundles in $E_{0}$, resp. $E$, have Chern class 1 .

Let $\bar{\partial}_{b}$ denote the tangential Cauchy-Riemann operator on $M_{0}$; then the corresponding operator for $M$ is $\bar{\partial}_{b}+\lambda \partial_{b}$, with $\lambda$ a small section of the bundle $H^{1,0} M_{0} \otimes\left(H^{0,1} M_{0}\right)^{*}$. By "small" we shall mean that $\|\lambda\|_{s}$ is small for some odd $s=7,9, \ldots$.

According to Lemma 7.1 there is a smooth diffeomorphism $F_{0}: \bar{E}_{0} \rightarrow \bar{D}_{0} \subset$ $\mathbb{P}_{2}$ (with $D_{0}$ denoting the pseudoconcave component of $\mathbb{P} \backslash M_{0}$ ), which is holomorphic on $E_{0}, F_{0}$ is the identity on $M_{0}$ and maps $C_{0}$ to the line at infinity $\mathbb{P}_{1}(\infty)$.

Assume now that, as in Theorem 4.5, $M$ bounds a strictly pseudoconvex surface $\bar{S}$. We have to show that there is a CR embedding $M \rightarrow \mathbb{C}^{2}$ close to $\left.F_{0}\right|_{M_{0}}$.

Let us attach $\bar{S}$ to $\bar{E}$ along their common boundary $M$. We obtain a compact complex surface $X$ without boundary. $X$ contains a biholomorphic image of $\mathbb{P}_{1}, C$, and the normal bundle of $C$ in $X$ is the hyperplane section bundle. $\bar{E}$ comes with a continuous function $v: \bar{E} \rightarrow[0, \infty]$, smooth and strictly plurisubharmonic on $\bar{E} \backslash C,\left.v\right|_{M}=0,\left.v\right|_{C}=\infty$ (see the proof of Theorem 6.5). From this $v$ we can construct a smooth exhaustion function $u: X \backslash C \rightarrow \mathbb{R}$, strictly plurisubharmonic outside a compact set $(\bar{S})$. Therefore the compact subvarieties in $X \backslash C$ can be blown down (see [11]), and we obtain a compact normal space $X^{\prime} \supset C$ such that $X^{\prime} \backslash C$ is Stein. The normal bundle of $C$ in $X^{\prime}$ is the same as before. In this situation a theorem due to Morrow and Rossi applies and yields that $X^{\prime}$ is biholomorphic to $\mathbb{P}_{2}$ (see [27]). Moreover, the proof there yields a biholomorphism that carries $C$ to $\mathbb{P}_{1}(\infty)$.

Now the compact subvarieties in $X \backslash C$ cannot intersect $\bar{E}$ (by virtue of the maximum principle applied to $v$ ), so $\bar{E} \subset X^{\prime}$, and the biholomorphism $X^{\prime} \rightarrow$ $\mathbb{P}_{2}$ restricts to a biholomorphism $F: \bar{E} \rightarrow \mathbb{P}_{2}$. The pullback of the hyperplane section bundle $\left[\mathbb{P}_{1}(\infty)\right] \rightarrow \mathbb{P}_{2}$ by $F$ is the bundle $[C] \rightarrow \bar{E}$. If homogeneous 
coordinates $\left(Z_{0}: Z_{1}: Z_{2}\right)$ are introduced on $\mathbb{P}_{2}$ so that $\mathbb{P}_{1}(\infty)=\left\{Z_{0}=0\right\}$, then $Z_{0}, Z_{1}, Z_{2}$ are sections of $\left[\mathbb{P}_{1}(\infty)\right]$ and pull back to sections $F^{0}, F^{1}, F^{2}$ of $[C]$. On $M, F_{0} \neq 0$ so the functions $f^{j}=F^{j} / F^{0}(j=1,2)$ are CR and define an embedding of $M$ into $\mathbb{C}^{2}$.

Similarly, with $M, F$ replaced by $M_{0}, F_{0}$ we obtain sections $F_{0}^{j}$ of $\left[C_{0}\right]$ and CR functions $f_{0}^{j}$ on $M_{0}$; in fact $\left(f_{0}^{1}, f_{0}^{2}\right)=\mathrm{id}_{M_{0}}$. Since $f_{0}^{j} F_{0}^{0}$ extends to a holomorphic section of $\left[C_{0}\right]$ we have $f_{0}^{j} \in L_{-}^{2}\left(M_{0}\right) \cap C^{\infty}\left(M_{0}\right) \quad(j=1,2)$. In the same way

$$
f^{j} \in L_{-}^{2}(M) \cap C^{\infty}(M)=L_{-}^{2}\left(M_{0}\right) \cap C^{\infty}\left(M_{0}\right), \quad j=1,2 .
$$

With the mapping $\omega$ introduced in $\S 11$ but with $M_{0}$ rather than $M$,

$$
\omega: C^{1}\left(M_{0}\right) \cap L_{-}^{2}\left(M_{0}\right) \rightarrow H^{0}\left(\left.\left[C_{0}\right]\right|_{C_{0}}\right) \oplus \mathbb{C},
$$

we have

Proposition 13.1. $\omega(1), \omega\left(f_{0}^{1}\right)$, and $\omega\left(f_{0}^{2}\right)$ are linearly independent. Also $\omega(1), \omega\left(f^{1}\right)$, and $\omega\left(f^{2}\right)$ are independent.

Proof. Obviously it suffices to prove that $\omega(1), \omega\left(f^{1}\right), \omega\left(f^{2}\right)$ are independent (since $M=M_{0}$ is permitted). Now assume that with complex numbers $c_{0}, c_{1}, c_{2}$ we have $\omega\left(c_{0}+c_{1} f^{1}+c_{2} f^{2}\right)=0$. Put $\Phi=c_{0}+c_{1} f^{1}+c_{2} f^{2}$. By Theorem $12.6\|\Phi\|_{s} \prec\left\|\bar{\partial}_{b} \Phi\right\|_{s-1}$. This implies $\left\|\partial_{b} \Phi\right\|_{s-1} \prec\left\|\bar{\partial}_{b} \Phi\right\|_{s-1}$, so that if $\|\lambda\|_{s}$ is sufficiently small

$$
\|\Phi\|_{s} \prec\left\|\bar{\partial}_{b} \Phi+\lambda \partial_{b} \Phi\right\|_{s-1}=0,
$$

since $\Phi$ is $\mathrm{CR}$ on $M$. Hence $c_{0}=c_{1}=c_{2}=0$.

Observe that

$$
\operatorname{dim} H^{0}\left(\left.\left[C_{0}\right]\right|_{C_{0}}\right)=\operatorname{dim} H^{0}\left(\left.\left[\mathbb{P}_{1}(\infty)\right]\right|_{P_{1}(\infty)}\right)=2
$$

(since the latter space is spanned by $Z_{1}, Z_{2}$ ). Thus $\omega$ takes values in a threedimensional space, so there is a matrix $A=\left(a_{j k}\right) \in \mathrm{GL}(3, \mathbb{C})$ such that, putting $f^{0}=f_{0}^{0}=1$,

$$
\omega\left(f_{0}^{j}\right)=\sum_{k=0}^{2} a_{j k} \omega\left(f^{k}\right), \quad j=0,1,2 .
$$

Define

$$
g^{j}=\sum_{k=0}^{2} a_{j k} f^{k} \in L_{-}^{2}(M) \cap C^{\infty}(M), \quad j=0,1,2 .
$$

Then $g^{j}-f_{0}^{j} \in \operatorname{Ker} \omega$. Also $g^{j}$ are CR functions on $M$; i.e., $\bar{\partial}_{b} g^{j}+\lambda \partial_{b} g^{j}=$ 0 . Hence, by Theorem 12.6

$$
\left\|g^{j}-f_{0}^{j}\right\|_{s} \prec\left\|\bar{\partial}_{b} g^{j}\right\|_{s-1} \prec\|\lambda\|_{s}\left\|\partial_{b} g^{j}\right\|_{s-1} .
$$

Therefore

$$
\left\|\partial_{b} g^{j}\right\|_{s-1} \prec\left\|g^{j}\right\|_{s} \prec\left\|f_{0}^{j}\right\|_{s}+\|\lambda\|_{s}\left\|\partial_{b} g^{j}\right\|_{s-1},
$$


and so, for $\|\lambda\|_{s}$ sufficiently small $\left\|\partial_{b} g\right\|_{s-1} \prec\left\|f_{0}^{j}\right\|_{s}$. Plugging this into (13.1), we get that $\left(g^{1}, g^{2}\right)$ defines a CR embedding of $M,\|\|_{s}$ close to $\left(f_{0}^{1}, f_{0}^{2}\right)$, and this proves Theorem 4.5.

Tracking down the constants in the above arguments we obtain the following quantitative version of Theorem 4.5:

Theorem 13.1. Let $\ell \geq 1$ be an integer. If $M_{0}$ is the boundary of a strictly linearly convex domain in $\mathbb{C}^{2}$ and $M$ is sufficiently close to $M_{0}$ in the $C^{72 \ell+609}$ norm and bounds a strictly pseudoconvex surface then $M$ admits an embedding into $\mathbb{C}^{2}, C^{\ell}$-close to the embedding $M_{0} \subset \mathbb{C}^{2}$.

14. On the spectrum of the boundary Laplacian. Let $M_{0}$ be an embeddable CR manifold, $\bar{\partial}_{b}$ the tangential Cauchy-Riemann operator on it. Picking a smooth metric on $M_{0}$, the adjoint $\bar{\partial}_{b}^{*}$ and $\square_{M_{0}}=\bar{\partial}_{b}^{*} \partial_{b}$ can be defined. If $M$ is a small perturbation of $M_{0}, \square_{M}$ can be defined similarly. $\square_{M}$ is a positive selfadjoint operator on $L^{2}(M)$. Because $M_{0}$ is embeddable, the spectrum Spec $\square_{M_{0}}$ consists of 0 (corresponding to CR functions) and a sequence of eigenvalues converging to $\infty$. The same holds for $\operatorname{Spec} \square_{M}$ if $M$ is embeddable while, for nonembeddable $M, \operatorname{Spec} \square_{M}$ will contain a sequence $\lambda_{j}>0$ tending to 0 : from the kernel of $\square_{M_{0}}$ infinitely many vectors split off to deform into eigenvectors of $\square_{M}$ corresponding to positive eigenvalues. All this is explained in [7].

The following question arises here. When $M$ is an embeddable perturbation of $M_{0}$, can (finitely many) vectors split off from $\operatorname{Ker} \square_{M_{0}}$ at all, or does $\operatorname{Ker} \square_{M_{0}}$ simply deform into $\operatorname{Ker} \square_{M}$ ? This question arose in [7], where the authors studied the space of embeddable CR manifolds. They conjectured that when $M_{0}=S^{3}$, no splitting off occurs, which would have the consequence that in the space of all perturbations of $S^{3}$ the space of embeddable perturbations is a closed set. Our stability Theorems 4.5 and 13.1 imply that their conjecture is true, even if $S^{3}$ is replaced by any strictly linearly convex hypersurface in $\mathbb{C}^{2}$ :

Theorem 14.1. Let $M_{0} \subset \mathbb{C}^{2}$ be the boundary of a strictly linearly convex domain. Then there are positive numbers $k, \varepsilon$ such that if $M$ is an embeddable perturbation of $M_{0}$ whose $C^{k}$ distance to $M_{0}$ is sufficiently small, then

$$
(0, \varepsilon) \cap \operatorname{Spec} \square_{M}=\varnothing .
$$

In fact, $k$ is independent of $M_{0}$.

Proof. Kohn, e.g. in [18], proves that any positive eigenvalue $\lambda$ of $\square_{M}$ satisfies $\lambda>c(M)>0$, if $M \subset \mathbb{C}^{2}$ is the boundary of a pseudoconvex domain. $c(M)$ can be computed using finitely many derivatives of a defining function of $M$. Thus Theorem 14.1 follows from Theorem 4.5.

Remark 14.2. The value of $k$ in the above theorem is of some interest. To actually compute it the approach in [18] may be too general. (It treats weakly pseudoconvex hypersurfaces, too). In [26] Ligocka calculates that the Bergman 
projection on a strictly pseudoconvex domain $G \subset \mathbb{C}^{2}$ is bounded in Hölder norms of order $\alpha \leq k$ once the domain is $C^{k+4}$ smooth. This suggests that $c(M)$ above, which is related to a Sobolev norm of order $\frac{1}{2}$ of a "Bergman projection" on forms, can be expressed through 5 derivatives of the defining function of $M$. This would yield $k=72 \cdot 5+609=969$.

\section{ACKNOWLEDGMENTS}

This paper owes a lot to ideas of J. Bland and T. Duchamp. They suggested that extremals of the Kobayashi metric on domains in $\mathbb{C}^{n}$ should be given a characterization purely in terms of the CR geometry of the boundary, and that this characterization should be used to decide embeddability of CR manifolds. It turned out that the emphasis had to be shifted from the Kobayashi metric to circle actions, but the importance of the former in this work cannot be overstated, and is evident in $\S \S 7$ and 8.

We are also very grateful to D. Catlin and J. Lee for several interesting discussions concerning some of the problems considered here.

We are indebted to S. Bell for calling our attention to Ligocka's paper.

\section{REFERENCES}

1. M. S. Baouendi, L. P. Rothschild, and F. Treves, CR structures with group action and extendability of $C R$ functions, Invent. Math. 82 (1985), 359-396.

2. D. Barrett, A remark on the global embedding problem for three-dimensional CR manifolds, Proc. Amer. Math. Soc. 102 (1988), 888-892.

3. E. Bedford and B. A. Taylor, The Dirichlet problem for a complex Monge-Ampère equation, Invent. Math. 37 (1976), 1-44.

4. J. Bland and T. Duchamp, Moduli for pointed convex domains, Invent. Math. 104 (1991), 61-112.

5. L. Boutet de Monvel, Intégration des equations de Cauchy-Riemann induites formelles, Séminaire Goulaoic-Lions-Schwartz, Exposé IX (1974-1975).

6. D. Burns, Global behavior of some tangential Cauchy-Riemann equations, Partial Differential Equations and Geometry (Proc. Conf., Park City, Utah), Marcel Dekker, New York 1979.

7. D. M. Burns and C. L. Epstein, Embeddabililty for three-dimensional CR-manifolds, J. Amer. Math. Soc. 3 (1990), 809-841.

8. H. Cartan, Quotient d'un espace analytic par un group d'automorphismes, Algebraic Geometry and Topology: A Symposium in honor of S. Lefschetz, Princeton University Press, Princeton, NJ, 1957, pp. 90-102.

9. Y. Cheng and J. Lee, personal communication.

10. G. B. Folland and E. M. Stein, Estimates for the $\bar{\partial}_{b}$ complex and analysis on the Heisenberg group, Comm. Pure Appl. Math. 27 (1974), 429-522.

11. H. Grauert, Über Modifikationen und exzeptionelle analytische Mengen, Math. Ann. 146 (1962), 331-368.

12. R. Harvey and L. B. Lawson, On the boundaries of complex analytic varieties. I, II, Ann. of Math. (2) 102 (1975), 233-290; 106 (1977), 213-238.

13. H. Jacobowitz and F. Treves, Non-realizable CR structures, Invent. Math. 66 (1982), 231249.

14. G. K Kiremidjian, A direct extension method for CR structures, Math. Ann. 242 (1979), $1-19$. 
15. S. Kobayashi, Hyperbolic manifolds and holomorphic mappings, Marcel Dekker, New York, 1970.

16. K. Kodaira, Complex manifolds and deformation of complex structures, Springer-Verlag, New York-Berlin-Heidelberg-Tokyo, 1986.

17. J. J. Kohn, Harmonic integrals on strongly pseudo-convex manifolds I, II, Ann. of Math. (2) 78 (1963), 112-148; 79 (1964), 450-472.

18. The range of the tangential Cauchy-Riemann operators, Duke Math. J. 53 (1986), 525-545.

19. A. Korányi and H. M. Reimann, Quasiconformal mappings on the Heisenberg group, Invent. Math. 80 (1985), 309-338.

20. __ Quasiconformal mappings on CR manifolds, Complex Geometry and Analysis: Proceedings of the international symposium in honour of Edoardo Vesentini, Lecture Notes in Math., vol. 1422, Springer, Berlin-Heidelberg-New York, 1990.

21. L. Lempert, La métrique de Kobayashi et la représentation des domaines sur la boule, Bull. Soc. Math. France 109 (1981), 427-474.

22. __ Intrinsic distances and holomorphic retracts, Proc. Conf. Complex Analysis and Applications '81 Sofia, Bulg. Acad. Sci., 1984, pp. 341-364.

23. gularity, Math. Ann. 263 (1983), 515-532.

24. Symmetries and other transformations of the complex Monge-Ampere equation, Duke Math. J. 52 (1985), 869-885.

25. _ A precise result on the boundary regularity of biholomorphic mappings, Math. Z. 193 (1986), 559-579.

26. E. Ligocka, The Hölder continuity of the Bergman projection and proper holomorphic mappings, Studia Math. 80 (1984), 89-107.

27. J. Morrow and H. Rossi, Some general results on equivalence of embeddings, Recent Developments in Several Complex Variables, Princeton Univ. Press, Princeton, N.J., 1981.

28. J. Moser, On the volume element on a manifold, Trans. Amer. Math. Soc. 129 (1965), 286-294.

29. L. Nirenberg, On a problem of Hans Lewy, Uspekhi Mat. Nauk 29 (1974), 241-251.

30. P. Orlik, Seifert manifolds, Lecture Notes in Math., vol. 291, Springer-Verlag, BerlinHeidelberg-New York, 1972.

31. G. Patrizio, Parabolic exhaustions for strictly convex domains, Manuscripta Math. 47 (1984), 271-309.

32. H. Rossi, Attaching analytic spaces to an analytic space along a pseudo-concave boundary, Proc. Conf. Complex Analysis (Minneapolis), Springer-Verlag, Berlin-Heidelberg-New York 1965.

33. H. L. Royden, Remarks on the Kobayashi metric, Several Complex Variables II (Proc. Internat. Conf., Univ. of Maryland, College Park, Md., 1970), Lecture Notes in Math., vol. 185, Springer-Verlag, Berlin-Heidelberg-New York, 1971.

34. E. H. Spanier, Algebraic topology, McGraw-Hill, New York, 1966.

35. N. Tanaka, A differential geometric study on strongly pseudoconvex manifolds, Lecture Notes in Math., Kyoto University, Kinokuniya Bookstore Co., Tokyo, 1975.

Department of Mathematics, Purdue University, West Lafayette, Indiana 47907

E-mail address: Lempert@gauss.math.purdue.edu 